OPEN ACCESS

Edited by:

Joyce Ann Guzik,

Los Alamos National Laboratory (DOE), United States

Reviewed by: Marc-Antoine Dupret, University of Liège, Belgium

Regner Trampedach, Space Science Institute, United States

*Correspondence: Da-run Xiong xiongdr@pmo.ac.cn

Specialty section: This article was submitted to

Stellar and Solar Physics,

a section of the journal Frontiers in Astronomy and Space Sciences

Received: 26 November 2018 Accepted: 28 October 2020 Published: 19 March 2021

Citation: Xiong D (2021) Convection Theory and Related Problems in Stellar Structure, Evolution, and Pulsational Stability II. Turbulent Convection and Pulsational Stability of Stars.

Front. Astron. Space Sci. 7:438870. doi: 10.3389/fspas.2020.438870

\section{Convection Theory and Related Problems in Stellar Structure, Evolution, and Pulsational Stability II. Turbulent Convection and Pulsational Stability of Stars}

\author{
Da-run Xiong * \\ Purple Mountain Observatory, Chinese Academy of Sciences, Nanjing, China
}

Using our non-local and time-dependent theory of convection and a fixed set of convective parameters $\left(C_{1}, C_{2} / C_{1}, C_{3}\right)=(0.70,0.50,3.0)$ calibrated against the Sun, the linear non-adiabatic oscillations for evolutionary models with masses 1-20 $\mathrm{M}_{\odot}$ are calculated. The results show that almost all the classical instability strips can be reproduced. The theoretical instability strips of $\delta$ Scuti and $\gamma$ Doradusvariables agree well with Kepler spacecraft observations. There is no essential difference in the excitation mechanism for $\delta$ Scuti and $\gamma$ Doradus stars. They are excited by the combined effects of the radiative к-mechanism and coupling between convection and oscillations. They represent two subgroups of a broader type of $\delta$ Scuti and $\gamma$ Doradus stars, located in the lower part of the Cepheid instability strip. $\delta$ Scuti is the $\mathrm{p}$-mode subgroup and $\gamma$ Doradus is the g-mode subgroup. The luminous variable red giants observed by MACHO and OGLE are low-order radial pulsators among low-mass red giant and asymptotic giant branch stars. The excitation and damping mechanism of oscillations for low-temperature stars is studied in detail. Convective flux and turbulent viscosity are consistent damping mechanisms. The damping effect of the convective enthalpy flux is inversely proportional to the frequency of the modes, so it plays an important role in stabilizing the low-order modes and defining the red edge of the Cepheid instability strip. The damping effect of turbulent viscosity reaches its maximum at $3 \omega \tau_{C} / 16 \sim 1$, where $\tau_{C}$ is the dynamic time scale of turbulent convection and $\omega$ is the angular frequency of the modes. Turbulent viscosity is the main damping mechanism for stabilizing the high-order modes of low-temperature variables. The turbulent pressure is, in general, an excitation mechanism; it reaches maximum at $3 \omega \tau_{c} / 4 \sim 1$, and it plays an important role for the excitation of red variables. Convection is not, in fact, a pure damping effect for stellar oscillations. The relative contributions of turbulent pressure, turbulent viscosity, and convective enthalpy flux for excitation and damping effects change with stellar parameters (mass, luminosity, effective temperature) and with the radial order and spherical harmonic degree of the oscillation mode; therefore, the combined effect of convection is sometimes damping, and sometimes the excitation of oscillations. Our research shows that, for low-luminosity red giants, the low-order modes are pulsationally stable, while the intermediate- and high-order modes are unstable. 
Toward higher luminosity, the range of unstable modes shifts gradually toward the lower order. All of the intermediate- and high-order modes become stable, and a few low-order modes become unstable for high-luminosity red giants. They show the typical pulsational characteristics of Mira-like variables. The variable red giants are, at least for the highluminosity RGs, self-excited. For red giants, the frequency of the maximally unstable modes predicted by our theory is similar to that given by the semi-empirical scaling relation.

Keywords: variables, interior-stars, convection-stars, oscillations-stars, evolution-stars

\section{INTRODUCTION}

Convection occurs within most stars. Convection brings about the transport and exchange of energy and momentum, and the mixing of matter in the stellar interior. Therefore, it strongly influences the structure, evolution, and pulsational stability of stars. Unfortunately, up to now no perfect convection theory has been accepted by the community. The most popularly applied convection theory is still the mixing-length theory (MLT, BohmVitense, 1958). The most obvious advantage of MLT is its straightforwardness in physical picture and simplicity of use. However, the MLT is not a dynamic theory following the hydrodynamic equations and turbulence theory, but is a phenomenological theory based on a simple analogy of turbulence with the kinetic theory of gas molecules. As such, it cannot give an exact description for turbulent convection, particularly for non-local and time-dependent convection. We developed a non-local and time-dependent theory of convection (Xiong et al., 1980; Xiong, 1981; Xiong, 1989; Xiong et al., 1997; Deng et al., 2006) based on hydrodynamic equations and turbulence theory in order to improve the treatment of overshooting in the calculation of stellar structure and evolution, and the treatment of coupling between convection and oscillations in the calculations of stellar oscillations. In Part I of this article, a non-local and time-dependent theory of convection developed by us was briefly described, and its applications in theoretical calculations of the structure of the solar convection zone, of massive star evolution, and of the lithium depletion in the atmospheres of late-type dwarfs were presented. Here in Part II, we illustrate its applications in the calculations of stellar oscillations. These applications are not only the primary motivation of our research on convective theory, but also the means for testing convection theory.

Stellar pulsation theory is being perfected thanks to the continuous work of generations of researchers for over 7 decades. Among others, we name four classical books by Ledoux and Walreven (1958), Cox (1980), Unno et al. (1989), and Aerts et al. (2011). They review the major research completed during that period of time. The remaining problems in stellar pulsation theory are almost all related to convection or non-linear theory. This paper is limited to the subjects of turbulent convection and the pulsational stability of stars.

Convection theory is still the most uncertain factor which prevents a clear understanding of oscillations in low- temperature stars. For low-temperature stars, dynamic coupling between convection and oscillations (through the exchange of momentum, i.e., turbulent pressure and turbulent viscosity) has at least as much, or possibly a larger effect than thermodynamic coupling (through the exchange of energy, i.e., convective enthalpy flux) on pulsational stability. The local description of convection has to be abandoned when turbulent pressure is taken into account in the equations of stellar structure, otherwise numerical calculations become unstable (Xiong, 1980). Our non-local and time-dependent theory of convection (Xiong, 1980; Xiong, 1981; Xiong, 1989; Xiong et al., 1997; Deng et al., 2006) is based on hydrodynamic equations and turbulence theory (Hinze, 1975); therefore, it has a more solid foundation in hydrodynamics, and provides a more precise description for the dynamic behavior of turbulent convection. Our formulation has the following advantages in the calculations of stellar oscillations:

(1) Our theory can be used for the treatment of both the thermodynamic (through the turbulent enthalpy flux) and dynamic coupling (through turbulent Reynolds stress, i.e., turbulent pressure and turbulent viscosity) between convection and oscillations in a self-consistent way.

(2) Our theory applies not only to radial oscillations but also to non-radial oscillations of stars in a self-consistent way, because our radiation-hydrodynamic equations are written in a general tensor format, which can deal not only with the exchange of energy and momentum in the radial direction, but also in the horizontal direction.

(3) In most calculations of non-adiabatic oscillations, the problematic rapidly oscillatory solutions are due to the local treatment method (Keeley, 1977; Baker and Gough, 1979; Gonczi and Osaki, 1980). Our time-dependent convection theory is non-local, so they have been effectively suppressed, and even completely removed (Xiong et al., 1998b; see also Figures 1-3 in the present paper).

(4) The pulsation stability is insensitive to convective parameters used. It is almost independent of the choice of convective parameters within a rather wide range (Xiong et al., 2015).

Linearizing our system of radiation-hydrodynamic equations for the calculation of stellar structure and oscillations in Eqs. (2), $\left(3^{\prime}\right),\left(4^{\prime}\right),(5)$ and Eqs. 18-21 in Part I, we produce the nonadiabatic radial and non-radial pulsation equations. They are more complex than other theories, they are a system of 

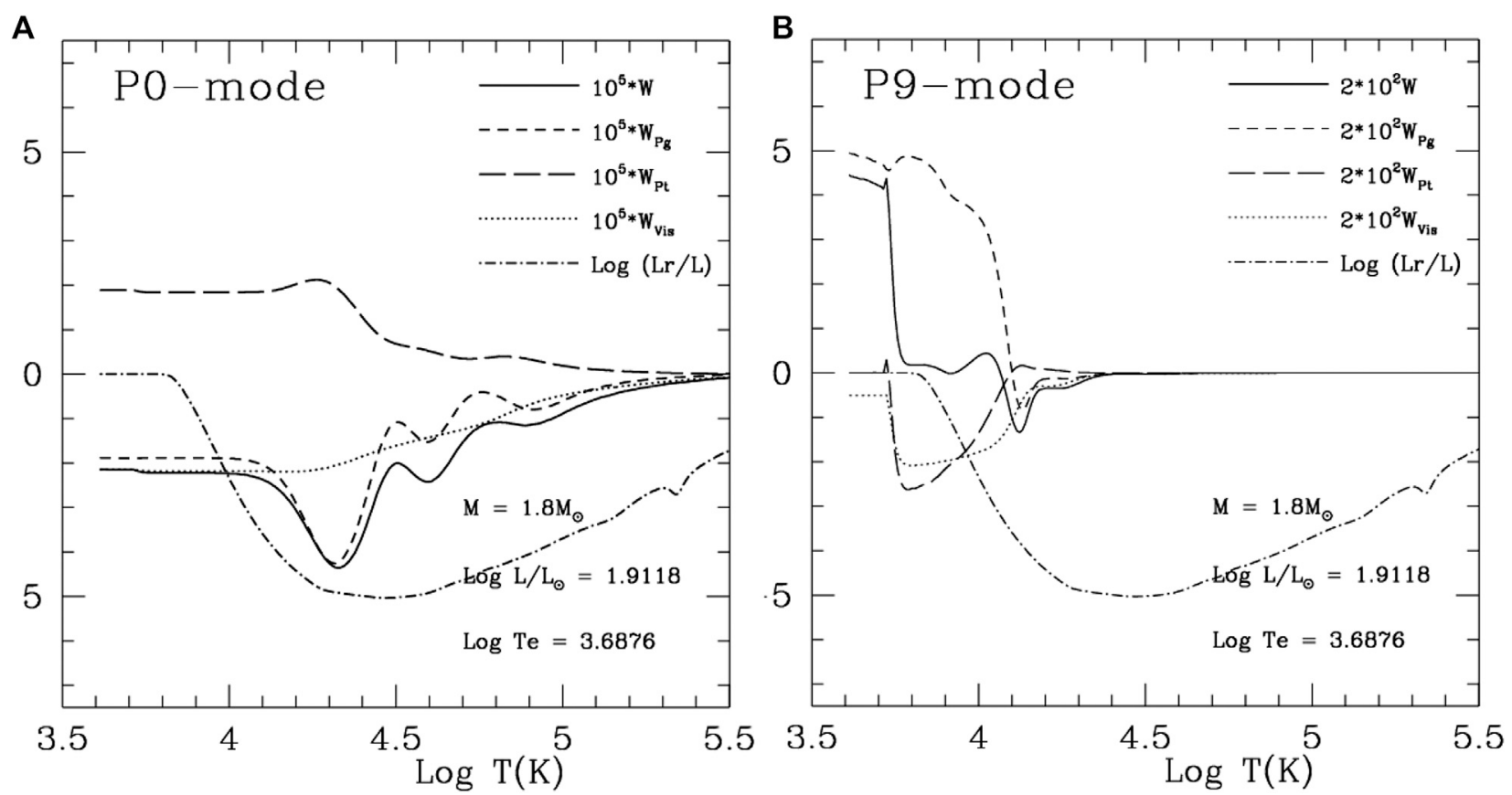

FIGURE 1 | Cumulative work $(W)$ and its gas pressure $\left(W_{p g}\right)$, turbulent pressure $\left(W_{P t}\right)$, and turbulent viscosity $\left(W_{\text {vis }}\right)$ components as functions of log $T$ for a lowluminosity red giant. $W=W_{p g}+W_{p t}+W_{\text {vis }}$ (see Eqs. 5-8). (A) P0-mode; (B) P9-mode.

differential equations of orders 10 and 16, respectively. For the adiabatic oscillation, the equations of energy conservation in Eq. $\left(4^{\prime}\right)$, and auto-correlation and cross-correlation for turbulent velocity and temperature in Eqs. 18-21 can be removed, the adiabatic radial and non-radial pulsation equations will be simplified to orders 2 and 6 . Therefore, the complexity and all the advantages mentioned above are due to our more accurate treatment of the convection problem. This should be the price for a more accurate convection theory, and it is worth it. The wave-scattering by inhomogeneous turbulent medium was neglected in the pulsation equations. Further investigation is needed.
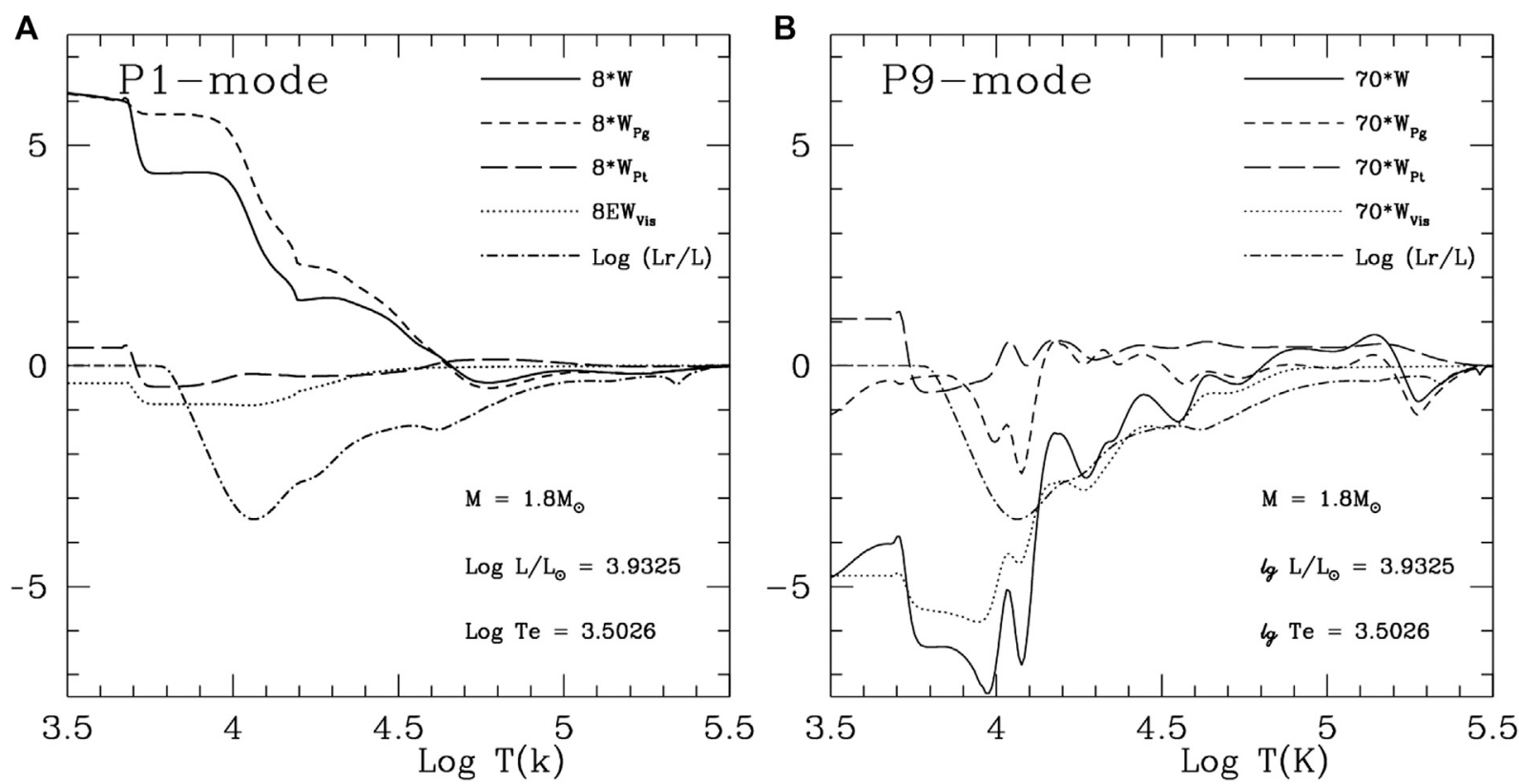

FIGURE 2 | The same as Figure 1, but for a high-luminosity red giant. 

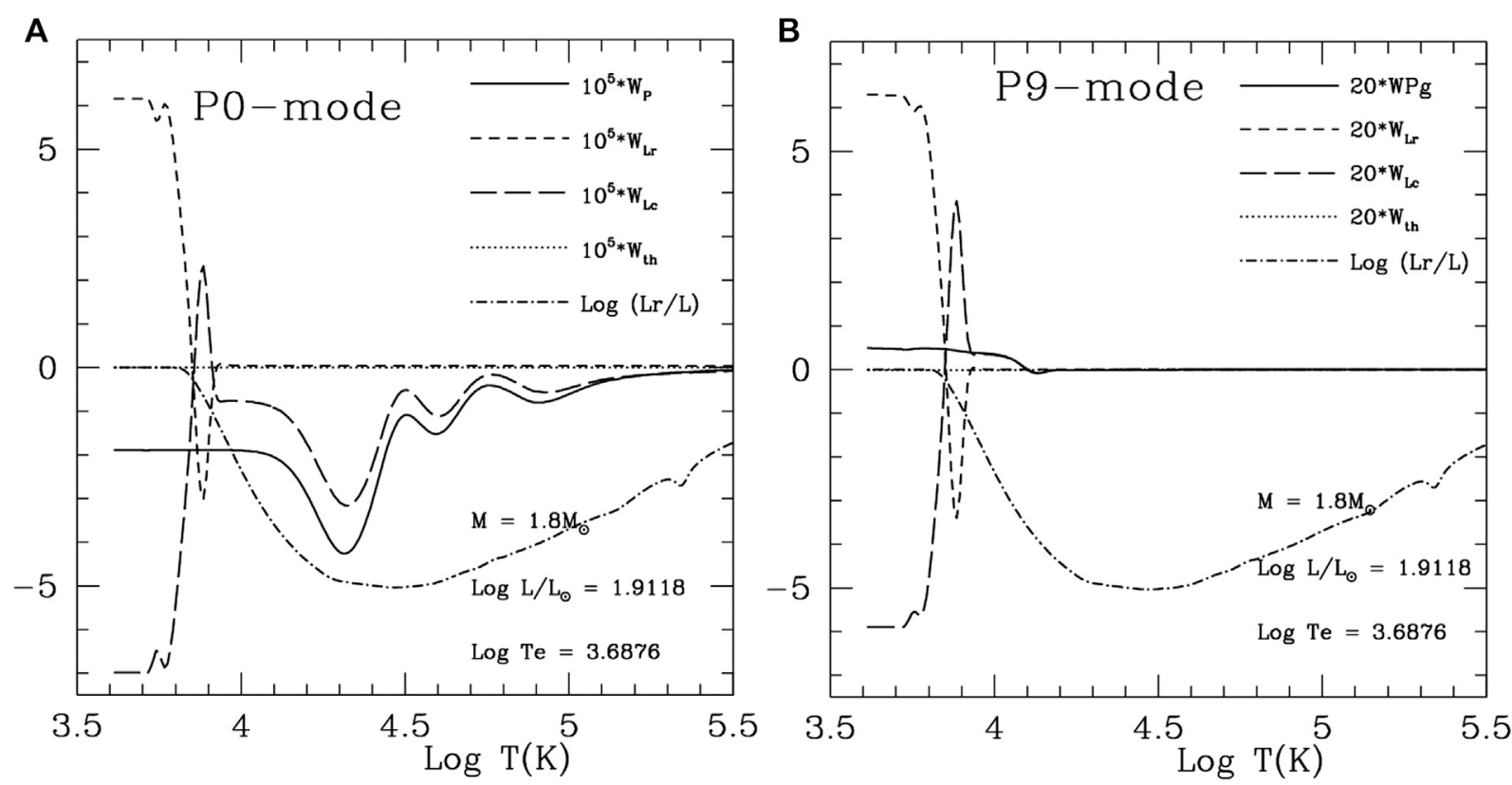

FIGURE 3 | Gas pressure $\left(W_{p g}\right)$, radiative flux $\left(W_{L r}\right)$, convective flux $\left(W_{L C}\right)$, and buoyancy work and turbulent dissipation components $\left(W_{t h}\right)$ of cumulative work as functions of $\log T$ for a low-luminosity red giant. $W_{p g}=W_{L r}+W_{L C}+W_{t h}$ (see Eqs. 10-13). (A) The same as in Figure 1A: P0-mode, (B) The same as in Figure 1B P9-mode.

In Theoretical Instability Strips, we present our theoretical instability strips in the H-R diagram. Recent progress in research of $\delta$ Scuti and $\gamma$ Doradus stars and luminous red variables is illustrated, respectively, in $\delta$ Scuti and $y$ Doradus Stars and Luminous Variable Red Giants. In Cumulative Work Analysis for the Excitation and Damping Mechanism, an analysis of the cumulative work for excitation and damping mechanisms is described in detail. In Excitation Mechanism for Variable Red Giants, the excitation mechanism of red variables is studied. A summary and discussion are found in Summary and Discussion.

\section{THEORETICAL INSTABILITY STRIPS}

By using our non-local and time-dependent theory of convection and a fixed set of convective parameters $\left(C_{1}, C_{2} / C_{1}, C_{3}\right)=$ $(0.70,0.50,3.0)$ calibrated against the Sun (Deng et al., 2006), we calculated the radial and non-radial non-adiabatic oscillations of evolutionary models with masses $1-20 M_{\odot}$ and the solar abundance $(Y=0.26, Z=0.017)$. In part II, all the evolutionary tracks ( $\mathrm{L}$ and $\mathrm{Te}$ ) are taken from Padova Tables by Bertelli et al. (2008, 2009). First, we calculated non-local convection envelope models along the Padova evolutionary tracks $(\mathrm{M}, \mathrm{L}, \mathrm{Te}, \mathrm{Y}, \mathrm{Z})$, and then calculated non-adiabatic oscillations of the non-local convection envelope models. The surface boundary was located at optical depth $\tau_{0}=10^{-3}$, and the bottom boundary was set deep enough for pulsational calculations, i.e., where the bottom temperature $T_{b}>6 \times 10^{6} \mathrm{~K}$ or the fractional radius $r_{b} / R_{0}<0.01$ is reached. A slightly modified version (Deng and Xiong, 2001) of the MHD equation of state (Däppen et al., 1988; Hummer and Mihalas, 1988; Mihalas et al., 1988) and OPAL opacity tables (Rogers and Iglesias, 1992) complemented by the low temperature opacity tables by Alexander and Ferguson (1994) were used.

Figure 4 shows the pulsationally unstable low-order non-radial $(l=2)$ p- (panel A) and g-modes (panel B) in the H-R diagram. Our research shows that the amplitude growth rate depends only on the frequency of the modes, and does not depend on the spherical harmonic degree (Xiong and Deng, 2010; Xiong et al., 2016). Figure 5 shows the pulsationally unstable low-order radial modes (colored, filled circles) in the H-R diagram. Each color is shifted a line-width in the $\mathrm{x}$ - $/ y$-direction, in order to be able to see each color. It can be seen from Figures 4, 5 that, by using a fixed set of convection parameters, almost all the classical instability strips, including the $\delta$ Cephei, $\delta$ Scuti and $\gamma$ Doradus (Xiong et al., 2016), $\beta$ Cephei (Deng and Xiong, 2001), slowly pulsating B-stars (SPB, Xiong and Deng, 2011), and LPV (Xiong et al., 1998a; Xiong et al., 2007; Xiong et al., 2018), can be reproduced. Their locations are marked in Figures 4, 5. $\beta$ Cephei and SPB stars are excited by the $\kappa$-mechanism from absorption by ions, peaking at $\mathrm{T} \sim 2 \times 10^{5} \mathrm{~K}$, so the width of these two instability strips depends sensitively on the metal abundance. $\beta$ Cephei and SPB stars in our Galaxy belong to the extreme population I, which have metal abundance higher than that of the Sun. This may explain why the widths of the theoretical instability strips of $\beta$ Cephei and SPB stars of the solar abundance in Figures 4, 5 are narrower than the observed ones in our Galaxy (belonging to extreme population I). Our theoretical instability strips, in fact, would agree well with the observations if the metal abundance is taken into account (Deng and Xiong, 2001; Tian et al., 2003). The blue dashed line and red solid line in 

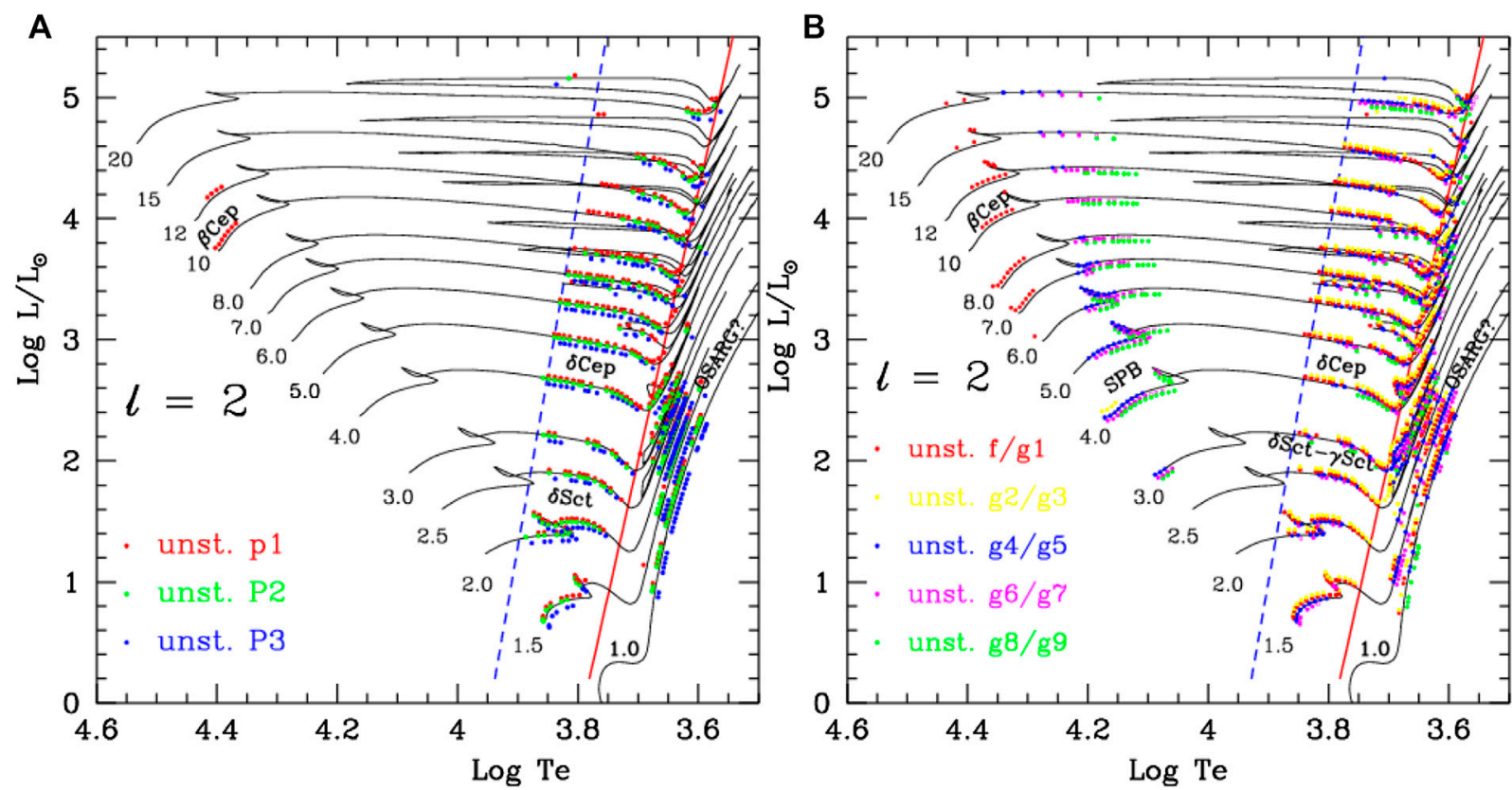

FIGURE 4 | Pulsationally unstable (colored, filled circles) low-degree (I = 2) non-radial low-order p-modes (panel a) and g-modes (panel b) in the H-R diagram for evolutionary models of $1-20 \mathrm{M}_{\odot}$ stars. Each color is shifted a line-width in the $x$-/y-direction, in order to be able to see each color. The blue dashed line and red solid line are, respectively, our theoretical blue and red edges of the Cepheid instability strip. The locations of the main types of variable stars are indicated.

Figures 4, 5 are, respectively, the blue and red edges of the Cepheid instability strip. The upper area of this instability strip is occupied by the classical Cepheids, and the lower area is occupied by the $\delta$ Scuti and $\gamma$ Doradus stars. The Cepheids and Cepheidlike stars are excited by the $\mathrm{k}$-mechanism in the hydrogen and helium ionization regions. The existence of the red edge (the red solid lines) is due to the convective damping through the convective enthalpy flux (see Cumulative Work Analysis for the Excitation and Damping Mechanism). There are many unstable stars in the lowtemperature region on the right-hand side of the red edge of the instability strip. They are pulsating red variables. They constitute the most abundant known population of variable stars, and yet we know little about them. Eggen (1973b) pointed out that all old disc population red giants with (R-I) $>0.9$ are variables. The visual amplitudes range from 0.01 (or less) to 10 magnitudes. The typical time-scale (period) goes from several hours to a few hundred days. Classification of these red variables based on the period, luminosity, and population data were made by Eggen (1972a); Eggen (1972b); Eggen 1973a; Eggen (1973b); Eggen (1975); Eggen (1977). Only the LRVs (luminous red variables, including Miras, irregular, semi-regular, and OGLE small amplitude variable red giants), were marked which are the best studied types among all the red variables. Comparing Figure 6 and Figure 7A with Figures 4A,B, 7B, it can be seen that the LRVs seem to favor radial oscillations. According to our theoretical calculations, the non-radial modes of low-mass $\left(\mathrm{M}<2 M_{\odot}\right)$ LRV luminous giants $\left(\log L / L_{\odot}>\sim 2.7\right)$ are stable (see Figure 7). This is only a theoretical prediction, the reality of which still needs to be confirmed. The details about the instability strips can be found by referring to our previous works cited in the parentheses following each of the variable types above and the related references cited in these papers. Studies of the excitation and damping mechanism of red variables are a main part of the present paper.

\section{$\delta$ SCUTI AND $\gamma$ DORADUS STARS}

Studies of $\delta$ Scuti and $\gamma$ Doradus stars have made significant progress in the past 2 decades thanks to the Kepler mission which has provided high-precision photometry in a continuous longtime series baseline for over 100,000 stars, among which about 1,500 new $\delta$ Scuti and $\gamma$ Doradus stars were discovered. This highprecision sample greatly enhanced our knowledge and offered opportunities for leaps in understanding of these two types of variables (Balona and Dziembowski, 2011; Balona et al., 2011). We calculated non-adiabatic oscillations of radial and low-degree non-radial modes for stellar evolutionary models with $M=1.4$ 3.0 $M_{\odot}$ and the solar abundance (Xiong et al., 2016). It can be seen from Figures 4, 5 that there is an obvious red edge for the loworder modes. The temperatures of the red edges in order from high to low are for radial modes, non-radial p-modes, and g-modes. Figure 6 shows the pulsationally stable (small, filled circles) and unstable modes (open circles) on the $n-\log T_{e}$ plane for the evolutionary models of a $2.0 M_{\odot}$ star, where $n$ is the radial order of the oscillation modes. $n>0$ are the p-modes and $n<0$ are the g-modes (Unno et al., 1989). It can be seen from Figure 6 that the instability strip changes with the radial order $n$. There are no red edges for intermediate-order $(20 \geq n \geq 6)$ radial and non- 


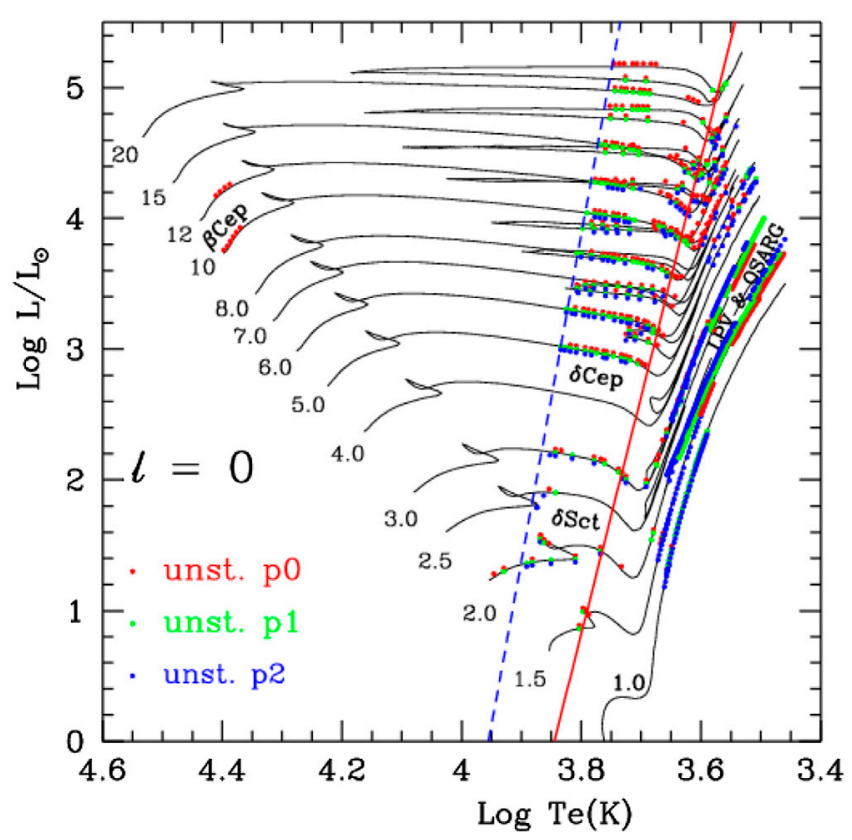

FIGURE 5|Pulsationally unstable radial low-order modes (colored, filled circles as labeled in the figure) in the $\mathrm{H}-\mathrm{R}$ diagram for evolutionary models of $1-20 \mathrm{M} \mathrm{P}_{\odot}$ stars. Each color is shifted a line-width in the $x$ - $/ y$-direction. The blue dashed line and red solid line are, respectively, our theoretical blue and red edges of the Cepheid instability strip. The locations of the main types of variable stars are indicated.

radial p-modes. This theoretical expectation of the existence of unstable intermediate-order modes in low-luminosity red giants still needs to be confirmed. This is because, in our theoretical calculations of the oscillations, the loss of coherence due to the scattering and refraction of acoustic waves caused by the inhomogeneous turbulent element, has been neglected;

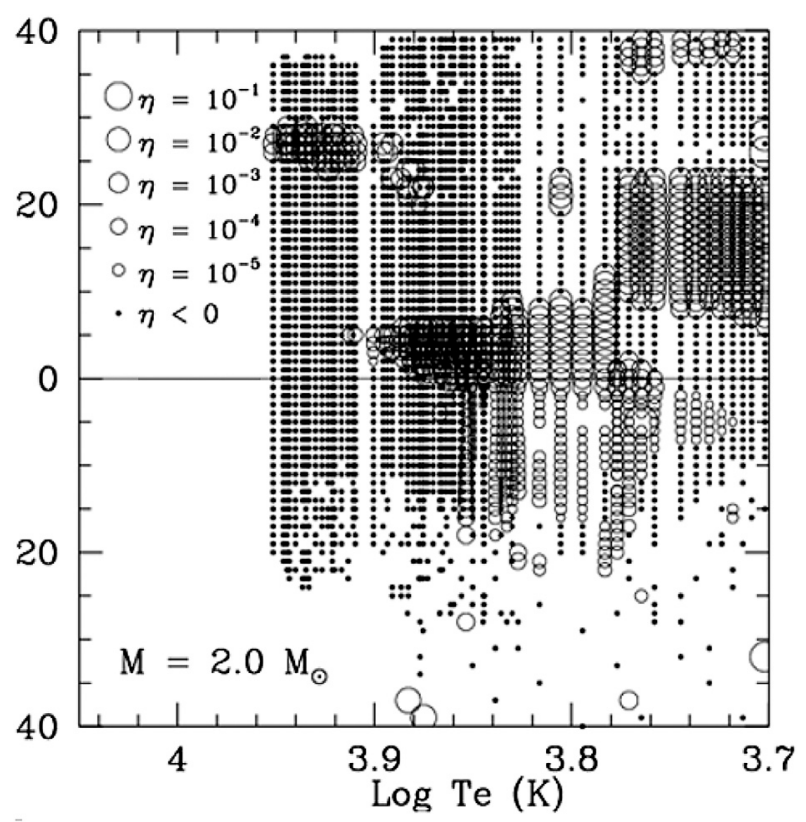

FIGURE 6 | Stable (small filled circles) and unstable (open circles) low-degree $(I=2)$ non-radial modes in the $\left(n-\right.$ log $\left.T_{e}\right)$ plane for evolutionary models of a $M=2.0 M_{\odot}$ star, where $n=n_{p}-n_{g}$ is the radial order of modes, and $n_{p}, n_{g}$ are, respectively, the node number of $\mathrm{p}$ - and g-modes (Unno et al., 1989 ). The size of the open circles is proportional to the logarithm of the amplitude growth rate of the modes. 

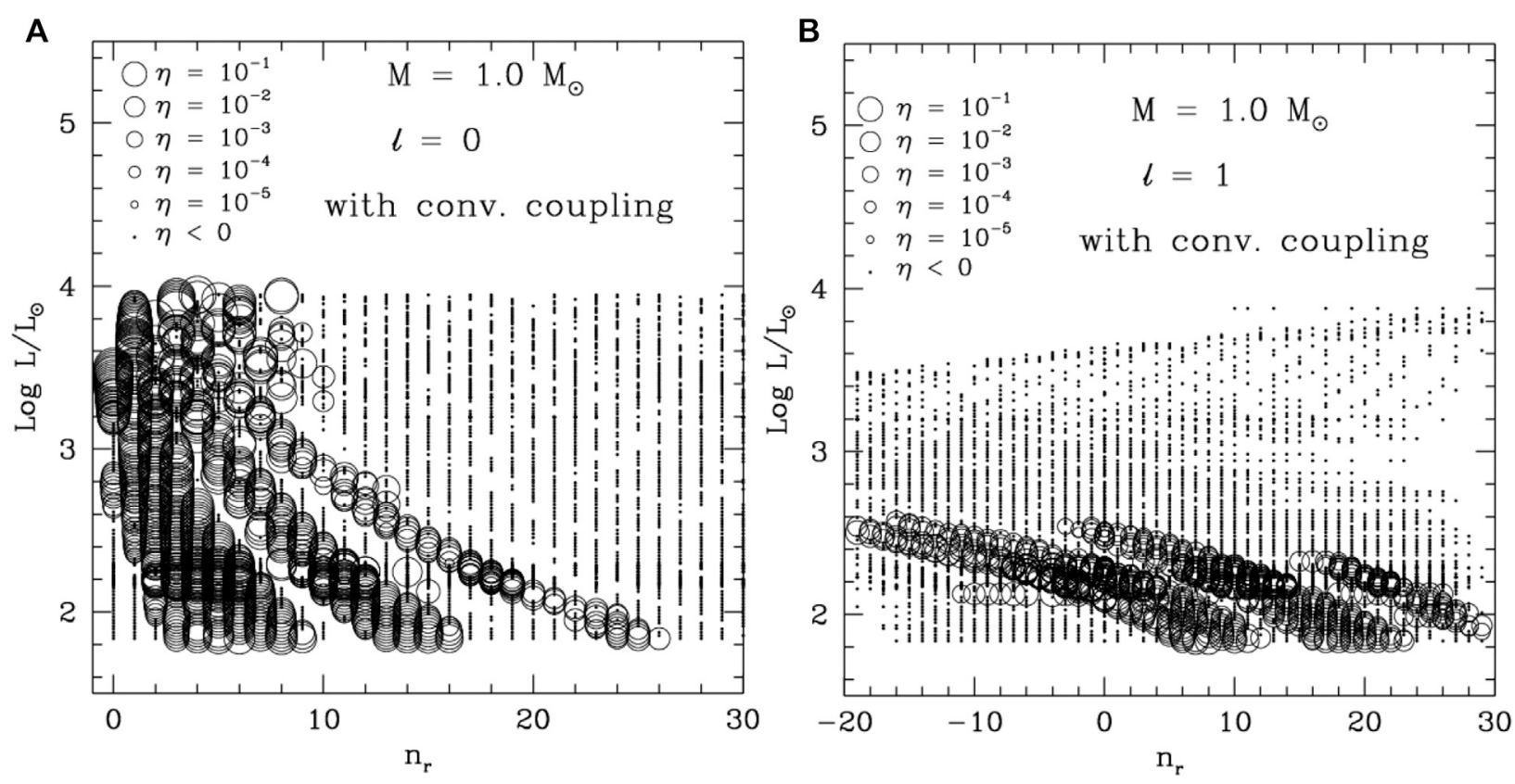

FIGURE 7 | (A) Pulsationally stable (small filled circles) and unstable (open circles) radial modes on the log $L / L_{\odot}-n_{r}$ plane for evolutionary AGB models of $M=1.0 M_{\odot}$. The size of the open circles are proportional to the logarithm of amplitude growth rate. (B) The same as $(\mathbf{A})$ but for low-degree $(I=1)$ non-radial modes. The abscissa $n_{r}=n_{p}-n_{g}$ is the radial order; $n_{p}$ and $n_{g}$ are, respectively, the node number for $\mathrm{p}$ - and g-modes.

therefore, the amplitude growth rate in our theory may be overestimated for the high-frequency modes.

$\gamma$ Doradus stars have long been thought to be a new type of variable, different from the classical Cepheid-like variables. They are excited by the so-called convective blocking mechanism (Guzik et al., 2000). This mechanism operates at the bottom of the convection zone. Therefore, it depends sensitively on the convection parameters used (Dupret et al., 2005; Pamyatnykh, 2000; Houdek, 2000; Grigahcene et al., 2010; Uytterhoeven et al., 2011; Hareter, 2012; Sarro et al., 2013). Our research (Xiong et al., 2016) shows that the excitation of $\gamma$ Doradus stars does not usually occur at the bottom of the convection zone, but rather in the outer layers of the star, in the ionization regions of hydrogen and helium. There is no essential difference between the excitation mechanisms of $\delta$ Scuti and $\gamma$ Doradus stars. Both result from the combined effects of the radiative $\kappa$-mechanism and coupling between convection and oscillations. The former plays a major role for the excitation of warm $\delta$ Scuti and $\gamma$ Doradus stars, while the latter is the main excitation and damping mechanism of cooler $\delta$ Scuti and $\gamma$ Doradus stars. We suggested that $\delta$ Scuti and $\gamma$ Doradus stars are two subgroups of a broader type of $\delta$ Scuti and $\gamma$ Doradus stars, located in the lower part of the Cepheid instability strip. $\delta$ Scuti stars form the p-mode subgroup, while $\gamma$ Doradus stars form the g-mode subgroup. It can be seen from Figures 4-6 that the $\delta$ Scuti and $\gamma$ Doradus instability strips overlap partially in the H-R diagram, with the latter being slightly redder. The stars located in the overlap region are mostly $\delta \mathrm{Scuti} / \gamma$ Doradus hybrids. The connections among $\gamma$ Doradus, $\delta$ Scuti, and $\delta \mathrm{Scuti} / \gamma$ Doradus hybrids are very similar to those among RRab, RRc, and RRd stars (Xiong et al., 2016).

\section{LUMINOUS VARIABLE RED GIANTS}

Studies of luminous variable red giants have progressed significantly in the past 2 decades thanks to OGLE (Soszynski et al., 2004; Soszynski et al., 2005; Soszynski et al., 2007; Soszynski et al., 2009), MACHO (Wood, 2000), and similar projects. The existence of the red edge of the Cepheid instability strip is due to damping from thermodynamic coupling between convection and oscillations (through thermal energy exchange, namely convective enthalpy flux). Therefore, fora long time, convection was understood as a pure damping mechanism for the oscillations of stars. Why are there various red variables in the low-temperature area beyond the Cepheid instability strip? This is still an outstanding and disputed subject. Xiong et al. (1998a); Xiong and Deng, (2007) studied in detail the coupling between convection and oscillations, and showed that there is a Mira instability strip in the low-temperature and high-luminosity area of the H-R diagram. Turbulent pressure plays an important role in the excitation of luminous red variables. OGLE and MACHO observations not only greatly enhanced the database, but also offered the best opportunity for studies of the luminous variable stars. By using our non-local and timedependent theory of convection and the same set of convection parameters $\left(C_{1}, C_{2} / C_{1}, C_{3}\right)=(0.70,0.50,3.0)$ as in Theoretical Instability Strips and $\delta$ Scuti and $y$ Doradus Stars, we carried out calculations of radial and non-radial nonadiabatic oscillations for evolutionary models of RGs and AGBs with $M=0.8-2.0 M_{\odot}$ (Xiong et al., 2018). The evolutionary models, EOS, and OPAL opacities used are the same as in Theoretical Instability Strips and $\boldsymbol{\delta}$ Scuti and $\gamma$ Doradus Stars. 
We chose an initial chemical abundance for the LMC of $\mathrm{Y}=0.26, \mathrm{Z}=0.008$. Figure $8 \mathrm{~A}$ shows our theoretical periodluminosity diagram for low-order radial modes. It can be seen that the theoretical fundamental through fourth overtone modes fall approximately on the observed sequences C-A' of the OGLE luminous variable red giants (Soszynski et al., 2004; Soszynski et al., 2005). The abscissa of Figure 8A is the logarithmic period $\log P$ and the ordinate is the reddening-free Wessenheit index $W_{I}$ (Madore, 1982) defined as

$$
W_{I}=I-1.55(V-I)
$$

The observed sequences $\mathrm{A}^{\prime}, \mathrm{A}, \mathrm{B}, \mathrm{C}^{\prime}$, and $\mathrm{C}$ of luminous red variables in the LMC are shown by magenta, blue, green, cyan, and red points in Figure 8A using data from the OGLE-III catalog of LPVs in the LMC (Soszynski et al., 2004; Soszynski et al., 2005; Soszynski et al., 2009).

The sequences C- $\mathrm{A}^{\prime}$ are commonly interpreted as different pulsation modes. But there is disagreement on the modal assignment. Based on the period ratios, Takayama et al. (2013) suggested that they can be explained by radial and low-degree nonradial $(l=1$ and 2$)$ low-order $\mathrm{p}$-modes $(\mathrm{n}=0-4)$. Wood (2015), Wood (2019) reached a similar conclusion in order to explain the closely spaced periods within a sequence in the Petersen diagram. Trabucchi et al. (2017) re-examined this problem. This is a somewhat complex subject. Wood (2015) found obvious evidence of mass variations within a sequence and from one sequence to another, and a sequence may also contain more than one type of mode. Figure $\mathbf{8 B}$ shows our theoretical period-luminosity diagram for $l=2$ non-radial modes ( $\mathrm{p} 0$ through $\mathrm{p} 4$ modes). It can be seen that they locate the shortperiod region far from the observations of the OGLE luminous variable red giants, and all the non-radial modes of luminous red giants $\left(W_{I}<\sim 14.5\right)$ are stable. The theoretical period-luminosity relations for $l=1$ non-radial modes are similar. Therefore, we believe that the sequences $\mathrm{C}-\mathrm{A}^{\prime}$ represent the period-luminosity (P-L) relations of the low-order radial modes of low-mass RGB and AGB stars rather than the non-radial ones. Comparing theoretical $\mathrm{P}-\mathrm{L}$ relations (Figure 8A) and period ratios with the observed ones, we suggest a possible mode identification that the sequences $C, C^{\prime}$, $\mathrm{B}, \mathrm{A}$, and $\mathrm{A}^{\prime}$ are, respectively, the radial fundamental to fourth overtone. This identification is identical with that of Wood. It seems to agree well with the observed P-L relation in Figure 8A, but the period ratios do not entirely agree with the observations. Another possible identification is as follows: sequences $C$ and $C^{\prime}$ are still the radial fundamental and first overtone; sequence $B$ is not the P-L relation of a single mode type, but represents the P-L relations of the second and first overtones; sequences $\mathrm{A}$ and $\mathrm{A}^{\prime}$ are, respectively, the second and third overtones. The theoretical period ratios of the latter identification are more consistent with the observations than those of the former identification. However, they do not entirely agree with the observations. The insufficiency of observations may be, in my opinion, an important reason. The OGLE data used for frequency analysis spanned 10 years, but typically there were only 700-900 points for each star (Soszynski et al., 2004). This time coverage, obviously, cannot ensure a perfect frequency analysis for LPVs, which are, in general, semi-regular and multiperiodic. This problem needs further investigation.

Studies for luminous red variables have greatly advanced our understanding, but there are still many outstanding problems:

(1) What kinds of variables are the OGLE small amplitude variable red giants (OSARGs)? Why are they so different from Miras and semi-regular variables located in the sequences of C and C' (Soszynski et al., 2004)?

(2) Are there true oscillation modes with period ratios $\sim 1$ and $\sim 0$ in the observed Petersen diagram, or are these spurious modes due to insufficient observations (Soszynski et al., 2004)?

(3) It can be seen from Figures $4 A, B, 8 A, B, 7 A, B$, that all the non-radial modes are stable for low-mass $\left(\mathrm{M}<2 M_{\odot}\right)$ luminous $\left(\log L / L_{\odot}>\sim 2.7\right)$ giants. If this theoretical prediction is correct, this means that the luminous red giants prefer to oscillate radially. This needs to be verified.

\section{CUMULATIVE WORK ANALYSIS FOR THE EXCITATION AND DAMPING MECHANISM}

Cumulative work is a convenient and efficient way to investigate the excitation and damping mechanism. Not only can the magnitude be estimated quantitatively for each component of excitation and damping, but also the location of these components can be identified. We give a simple derivation of cumulative work. Linearizing the average equation of momentum conservation in Eq. 3 in part I and neglecting the molecular viscous term (it is far smaller than any other terms in the equation), we have,

$$
\omega^{2} \delta r^{i}=\frac{1}{\bar{\rho}} \nabla_{k}\left[g^{i k}\left(\bar{P}+\bar{\rho} x^{2}\right)^{\prime}+\left(\bar{\rho} \chi^{i k}\right)^{\prime}\right]+g^{i k} \nabla_{k} \Phi^{\prime},
$$

$\delta r^{i}$ here and $\delta r_{i}$ below are, respectively, the $i$ th inverse-variant (superscript) and covariant (subscript) components of the displacement vector. Multiplying Eq. 2 by $\delta r_{i}^{*} d M_{r} \quad\left(\delta r_{i}^{*}\right.$ is the conjugate value of $\delta r_{i}$ ), summing $\mathrm{i}$ from 1 to 3 , and then integrating with respect to $d M_{r}$ for the whole star, the integration of the left-hand side of Eq. 2 becomes

$$
\int_{0}^{M_{0}} \omega^{2} \delta r^{i} \delta r_{i}^{*} d M_{r}=2\left(1-\frac{\omega_{i}^{2}}{\omega_{r}^{2}}+2 i \frac{\omega_{i}}{\omega_{r}}\right) E_{k}
$$

where $\omega_{i}, \omega_{r}$ are, respectively, the imaginary and real part of the complex angular frequency $\omega=i \omega_{i}+\omega_{r}$, and $E_{k}$ is the total kinetic energy of an oscillation mode,

$$
E_{k}=\frac{\omega_{r}^{2}}{2} \int_{0}^{M_{0}} d \delta r^{i} \delta r_{i}^{*} M_{r}=\frac{\omega_{r}^{2}}{2} \int_{0}^{M_{0}}\left[\delta r^{2}+l(l+1) \delta r_{h} \delta r_{h}^{*}\right] d M_{r}
$$

where $\delta r$ and $\delta r_{h}$ are, respectively, the radial and horizontal components of the shift vector. $\eta=-2 \pi \omega_{i} / \omega_{r}$ is the amplitude growth rate per period. From the integration of the right-hand side of Eq. 2, we find the normalized cumulative work W: 

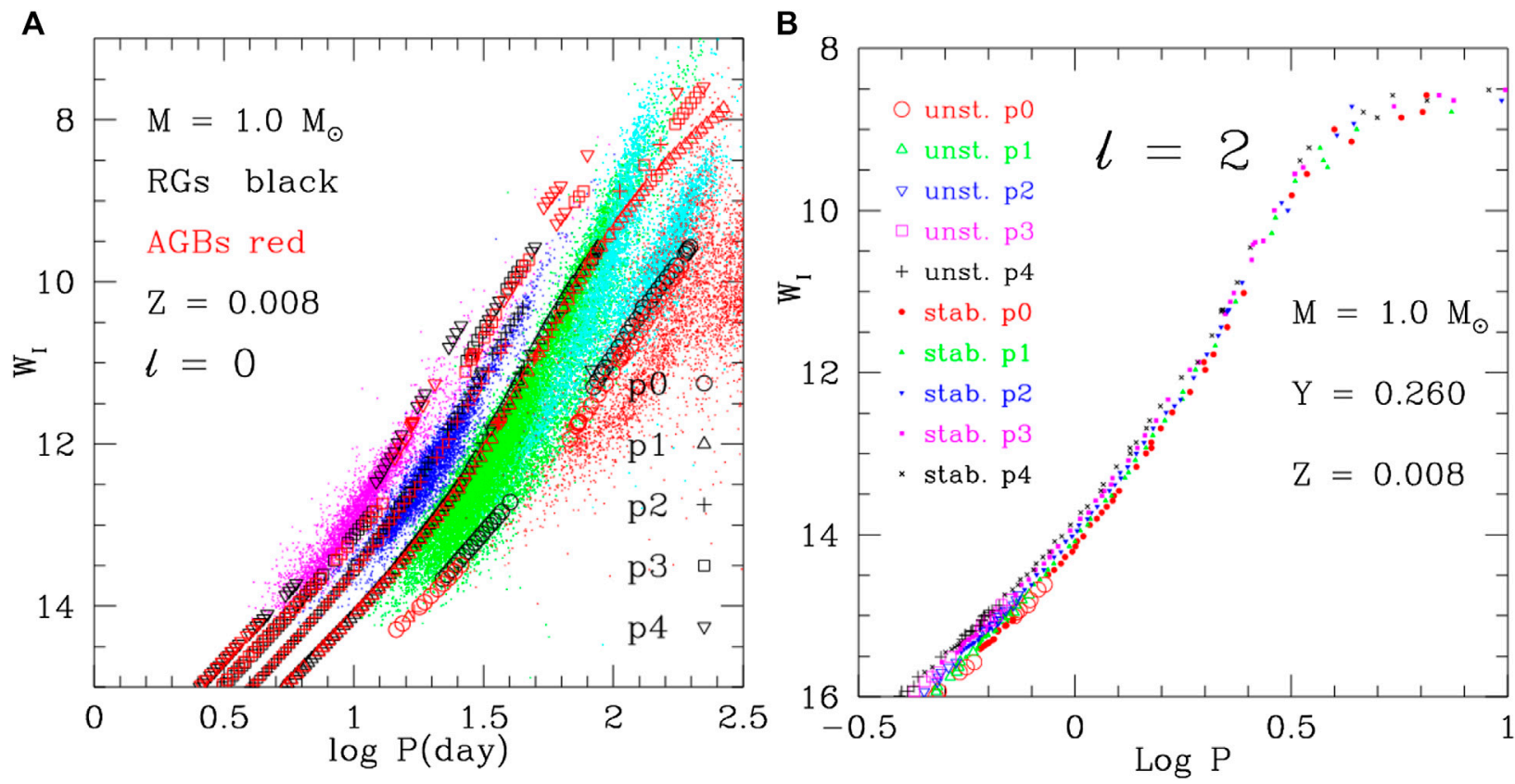

FIGURE 8|Period-luminosity diagram of low-order radial (panel a) and non-radial (panel b) modes for evolutionary models of RGs (black) and AGBs (red) with mass $M=1.0 M_{\odot}$. The open and filled circles, triangles, pluses, squares, and inverse triangles are, respectively, the unstable and stable fundamental through fourth overtone modes. The observed P-L sequences $A, A^{\prime}, B, C^{\prime}$, and $C$ are shown by magenta, blue, green, cyan, and red small points.

$$
\begin{aligned}
W_{\left(M_{r}\right)} & =\frac{\pi}{2 E_{k}} \int_{0}^{M_{r}} I_{m}\left\{-\left[\frac{\delta \bar{P}}{\bar{\rho}}+2 x^{2} \frac{\delta x}{x}\right] \frac{\delta \bar{\rho}^{*}}{\bar{\rho}}\right. \\
& \left.+\delta \chi_{1}^{1} \frac{d}{d \ln r}\left(\frac{\delta r^{*}}{r}\right)\right\} d M_{r} \\
= & W_{p g\left(M_{r}\right)}+W_{p t\left(M_{r}\right)}+W_{v i s\left(M_{r}\right) .}
\end{aligned}
$$

The value of $W_{\left(M_{r}\right)}$ at the surface of stars, $W_{\left(M_{0}\right)}$, is equal to the amplitude growth rate per period $\eta=-2 \pi \omega_{i} / \omega_{r}$, these agree with each other to within several percent in general. Here $W_{\left(M_{r}\right)}$ is the total cumulative work, and $W_{p g\left(M_{r}\right)}, W_{p t\left(M_{r}\right)}$, and $W_{v i s\left(M_{r}\right)}$ are, respectively, the gas pressure, turbulent pressure, and turbulent viscosity components of the cumulative work,

$$
\begin{gathered}
W_{p g\left(M_{r}\right)}=-\frac{\pi}{2 E_{k}} \int_{0}^{M_{r}} I_{m}\left\{\frac{\bar{P}}{\bar{\rho}} \frac{\delta \bar{P}}{\bar{P}} \frac{\delta \bar{\rho}^{*}}{\bar{\rho}}\right\} d M_{r}, \\
W_{p t\left(M_{r}\right)}=-\frac{\pi}{E_{k}} \int_{0}^{M_{r}} I_{m}\left\{x^{2} \frac{\delta x}{x} \frac{\delta \bar{\rho}^{*}}{\bar{\rho}}\right\} d M_{r}, \\
W_{v i s\left(M_{r}\right)}=\frac{\pi}{2 E_{k}} \int_{0}^{M_{r}} I_{m}\left\{\delta \chi_{1}^{1} \frac{d}{d \ln r}\left(\frac{\delta r^{*}}{r}\right)\right\} d M_{r},
\end{gathered}
$$

where $\delta \bar{P}, \delta \bar{\rho}, \delta x$, and $\delta \chi_{1}^{1}$ are, respectively, the Lagrangian perturbations of $\bar{P}, \bar{\rho}, x$, and $\chi_{1}^{1} \cdot \bar{\rho} x^{2}$ and $\bar{\rho} \chi^{i j}$ are, respectively, the isotropic (namely turbulent pressure) and anisotropic components of turbulent Reynolds stress. The variation of turbulent pressure $\rho x^{2}$ in the process of stellar oscillations always lags slightly behind the variation of density due to the inertia of turbulent kinetics. As a result, a positive Carnot cycle is formed in the $P_{t}-V=(1 / \rho)$ diagram during oscillations of a star, so the stochastic turbulent kinetic energy is converted into the coherent kinetic energy of stellar oscillations. Therefore, the turbulent pressure is, in general, an excitation mechanism of oscillations. The anisotropic component of Reynolds stress (see Eq. 15 below), $\rho \chi^{i j}$, on the other hand, converts the coherent pulsation kinetic energy into stochastic turbulent kinetic energy due to the shear effects of fluid motion, so the turbulent viscosity always has a damping effect on stellar oscillations. The gas pressure component $W_{p g}$ includes the contribution of both radiative and convective fluxes. Linearizing the thermal energy conservation equation in Eq. $4^{\prime}$ in Part I, it is not difficult to obtain

$$
\begin{aligned}
\frac{\delta \bar{P}}{\bar{P}}-\Gamma_{1} \frac{\delta \rho}{\bar{\rho}}= & \frac{\Gamma_{3}-1}{i \omega \bar{P}}\left\{\alpha V \frac{G M_{r}}{r^{2}}\left[\left(2+\frac{r^{3} \omega^{2}}{G M_{r}}\right) \frac{\delta r}{r}-\frac{\delta V}{V}\right]\right. \\
& +\delta\left(\frac{4 \sqrt{3} \eta_{e} G M_{r} \bar{\rho}}{3 C_{1} r^{2} \bar{P}} x^{3}\right)-\frac{1}{4 \pi r^{2} \bar{\rho}} \frac{d}{d r}\left(\delta L_{r}+\delta L_{c}\right. \\
& \left.\left.+\delta L_{t}\right)+\frac{l(l+1)}{r \bar{\rho}}\left(F_{r, h}^{\prime}+F_{c, h}^{\prime}+F_{t, h}^{\prime}\right)\right\},
\end{aligned}
$$

where $\Gamma_{1}-\Gamma_{3}$ are the adiabatic indexes introduced by Chandrasekhar (1939). $\left(\delta L_{r}, F_{r, h}^{\prime}\right),\left(\delta L_{c}, F_{c, h}^{\prime}\right)$, and $\left(\delta L_{t}, F_{t, h}^{\prime}\right)$, are, respectively, the radial Lagrange and horizontal Euler components of the radiative flux, convective enthalpy flux, and turbulent kinetic energy flux vector. The terms on the right side of Eq. 9 are the contribution of nonadiabatic effects. The first term in square brackets is the net loss of thermal energy due to buoyancy work, the second term is the gain of thermal energy due to turbulent dissipation, and the third and fourth terms are the net gain of thermal energy from the radiative and 


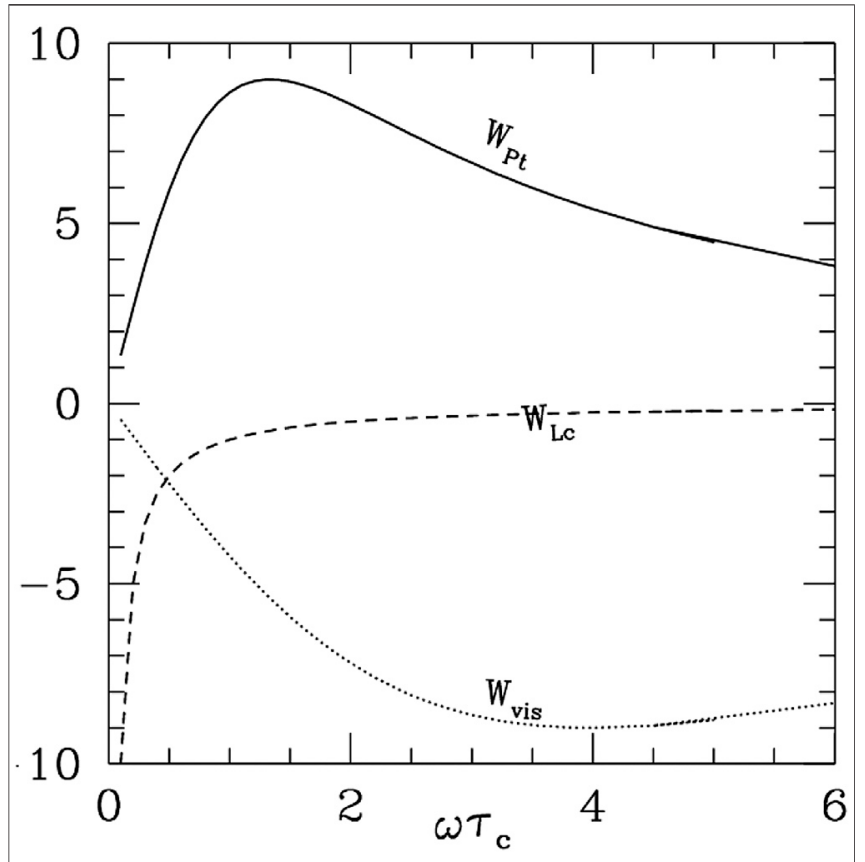

FIGURE 9 | Frequency dependence of the excitation and damping effects of turbulent pressure (solid line), turbulent viscosity (dotted line), and convective enthalpy flux (dashed line) on stellar oscillations. The ordinate is used in any unit.

convective enthalpy fluxes, and turbulent kinetic energy flux. Substituting Eq. 9 into Eq. 6, the contributions of radiative flux $\left(W_{L r\left(M_{r}\right)}\right)$, convective flux $\left(W_{L c\left(M_{r}\right)}\right)$, and buoyancy work and turbulent dissipation $\left(W_{\text {th }\left(M_{r}\right)}\right)$ can be separated:

$$
W_{p g\left(M_{r}\right)}=W_{L r(r)}+W_{L c(r)}+W_{t h(r)},
$$

where

$$
\begin{aligned}
& W_{L r\left(M_{r}\right)}=-\frac{\pi}{2 E_{k} \omega} \int_{0}^{M_{r}}\left(\Gamma_{3}-1\right) R_{e}\left\{\left[\frac{1}{4 \pi r^{2} \bar{\rho}} \frac{d \delta L_{r}}{d r}\right.\right. \\
& \left.\left.-\frac{l(l+1) L_{r, h}^{\prime}}{4 \pi r^{3} \bar{\rho}}\right] \frac{\delta \bar{\rho}^{*}}{\bar{\rho}}\right\} d M_{r}, \\
& W_{L c\left(M_{r}\right)}=-\frac{\pi}{2 E_{k} \omega} \int_{0}^{M_{r}}\left(\Gamma_{3}-1\right) R_{e}\left\{\left[\frac{1}{4 \pi r^{2} \bar{\rho}} \frac{d \delta L_{c}}{d r}\right.\right. \\
& \left.\left.-\frac{l(l+1) L_{c, h}^{\prime}}{4 \pi r^{3} \bar{\rho}}\right] \frac{\delta \bar{\rho}^{*}}{\bar{\rho}}\right\} d M_{r}, \\
& W_{t h\left(M_{r}\right)}=\frac{\pi}{2 E_{k} \omega} \int_{0}^{M_{r}}\left(\Gamma_{3}-1\right) R_{e}\left\{\left(\frac { G M _ { r } } { r ^ { 2 } } \alpha V \left[\left(2+\frac{r^{3} \omega^{2}}{G M_{r}}\right) \frac{\delta r}{r}\right.\right.\right. \\
& \left.\left.\left.-\frac{\delta V}{V}\right]+\delta\left(\frac{4 \sqrt{3} \eta_{e} G M_{r} \bar{\rho}}{C_{1} r^{2} \bar{P}} x^{3}\right)\right) \frac{\delta \bar{\rho}^{*}}{\bar{\rho}}\right\} d M_{r}
\end{aligned}
$$

The first term in the brackets of Eq. 13 is the buoyancy work, that is, the conversion rate from thermal energy into turbulent kinetic energy, so its contribution for $W_{t h\left(M_{r}\right)}$ is negative. This process occurs in the region of the low wave-number of turbulent spectrum. The second term represents the dissipation rate of turbulent kinetic energy, that is, the conversion rate from turbulent kinetic energy into thermal energy, so its contribution for $W_{t h\left(M_{r}\right)}$ is positive. This process occurs in the region of the high wave-number of turbulent spectrum. For a static star, these contrary transfer processes between thermal energy and turbulent energy will bring the turbulence to an equilibrium state. It can be known from Eq. 18 (in Part I) that the sum of these two terms is approximately zero for a static star. For a pulsating star, it is not zero, but it is still small in comparison with $W_{L r\left(M_{r}\right)}$ and $W_{L c\left(M_{r}\right)}$. After analysis, it is easy to understand the behavior of $W_{p g}\left(M_{r}\right)$ and its components $W_{L r(r)}, W_{L c(r)}$, and $W_{t h(r)}$ as illustrated in Figure 3.

Linearizing Eqs. (18) and (19) in Part I and neglecting the third-order correlation terms, we have

$$
\begin{gathered}
\delta x^{2} \approx \\
\frac{2}{3} \frac{1}{1+i \omega \tau_{c 1}}\left\{i \omega \tau_{c 1}\left[x^{2} \frac{\delta \bar{\rho}}{\bar{\rho}}-\chi_{1}^{1} \frac{d}{d \ln r}\left(\frac{\delta r}{r}\right)\right]\right. \\
\left.+\frac{G M_{r} \alpha V \tau_{c 1}}{r^{2}}\left[\frac{\delta V}{V}-\left(2+\frac{r^{3} \omega^{2}}{G M_{r}}\right) \frac{\delta r}{r}\right]\right\}, \\
\delta \chi_{1}^{1} \approx \frac{4}{3} \frac{1}{1+i \omega \tau_{c 2}}\left\{-i \omega \tau_{c 2}\left[\left(x^{2}+\chi_{1}^{1}\right) \frac{d}{d \ln r}\left(\frac{\delta r}{r}\right)+\frac{3}{2} \chi_{1}^{1} \frac{\delta r}{r}\right]\right. \\
\left.+\frac{G M_{r} \alpha V \tau_{c 2}}{r^{2}}\left[\frac{\delta V}{V}-\left(2+\frac{r^{3} \omega^{2}}{G M_{r}}\right) \frac{\delta r}{r}\right]\right\}, \quad \tau_{c 1}=\frac{3}{4} \tau_{c}, \\
\text { where } \\
\tau_{c 2}=\frac{C_{1} r^{2} \bar{P}}{\sqrt{3} \eta_{e} G M_{r} \bar{\rho} x}, \quad \tau_{c} .
\end{gathered}
$$

Eqs. 4-16 are our expressions for cumulative work in the general case of non-radial oscillations. They will reduce to the radial expression for $l=0$. It can be found from Eqs. 14-16 that the turbulent pressure and turbulent viscous components $W_{p t}$ and $W_{v i s}$ reach their maxima, respectively, at $\omega \tau_{c 1}=1$ and $\omega \tau_{c 2}=1$. It can be found from Eq. 11 that the convective flux component $W_{L c}$ always dampens stellar oscillations and is inversely proportional to the oscillation frequency of the modes; it plays an important role in stabilizing the low-order modes of low-temperature stars and defines the red edge of the instability strip.

Figure 9 shows the frequency dependences of the excitation and damping effects of turbulent pressure, viscosity, and convective enthalpy flux.

\section{EXCITATION MECHANISM FOR VARIABLE RED GIANTS}

There is a red edge for the Cepheid (and Cepheid-like) instability strip in the $\mathrm{H}-\mathrm{R}$ diagram due to convective damping, as mentioned in the previous section. Therefore, convection for a long time was thought of as a purely damping mechanism for stellar oscillations. Convection is not, in fact, a pure damping effect for stellar oscillations, as described in Cumulative Work Analysis for the Excitation and Damping Mechanism. The relative 
contributions of turbulent pressure, turbulent viscosity, and convective enthalpy flux for excitation and damping of modes change with stellar parameters (mass, luminosity, and effective temperature) and the pulsation frequency (or, alternatively, the radial order and spherical harmonic degree) of the mode. Therefore, the net effect of convection is sometimes to dampen, and sometimes to excite an oscillation mode. Figure 7 shows the pulsationally stable and unstable modes in the $\log L / L_{\odot}-n_{r}$ plane for the evolutionary models of red giants with $M=1.0 M_{\odot}\left(\log T_{e}<3.70\right)$, where $n_{r}$ is the radial order of the modes (Xiong et al., 2018). It can be seen that for lowluminosity red giants, the low-order modes are pulsationally stable, while the intermediate- and high-order modes are unstable. Toward high luminosity, the unstable modes move gradually toward the lower order. All of the intermediate- and high-order modes become stable, while a few low-order modes become unstable for high-luminosity red giants. Their pulsational characteristics are typical of Mira-like stars. The turbulent pressure plays an important role for the excitation of variable red giants. The excitation effect of turbulent pressure reaches its maximum at $\omega^{\omega} \tau_{C 1} \sim 1$. From Eq. 16, the frequency of the maximally unstable mode is

$$
v_{\text {max }} \sim \frac{M \bar{\rho} x}{C_{1} r^{2} P} \sim \frac{M T^{3.5} C_{s}}{L x}
$$

where $C_{s} \sim T^{1 / 2}$ is the adiabatic acoustic velocity, and $C_{s} / x$ is the Mach number of turbulence, which changes little from one star to another. Eq. 17 is, in fact, similar to the scaling relation. Figure 10 shows a comparison of the frequency of the maximally unstable mode predicted by our non-adiabatic oscillation calculations $v_{\text {Xiong }}$ (coherent excitation, abscissa) with $v_{\text {Bedding }}$ (ordinate) predicted by the scaling relation of stochastic excitation theory (Kjeldsen and Bedding, 1995; Stello et al., 2007). It can be seen that they agree well with each other. This means that it is impossible to distinguish these two excitation mechanisms based only on the frequency of the highest mode amplitude.

In the cumulative work analysis (Cumulative Work Analysis for the Excitation and Damping Mechanism) and in our theoretical calculations of non-adiabatic oscillations (Theoretical Instability Strips, $\delta$ Scuti and $\gamma$ Doradus Stars, Luminous Variable Red Giants), the radiative flux, convective thermodynamic (via the convective enthalpy flux), and dynamic coupling (via the turbulent Reynolds stress, i.e., turbulent pressure and turbulent viscosity) are taken into account in a self-consistent way. Our convection theory is a dynamic theory based on the hydrodynamic equations and turbulence theory. Compared with MLT, it has a more solid theoretical foundation in hydrodynamics. Therefore, it is expected to more accurately describe the dynamic behavior of turbulent convection, and it can produce more reliable results than MLT for classic variables and luminous red variables. However, for low-luminosity lowtemperature stars, such as the solar-like oscillators, the modes are high-degree and high-order radial and non-radial p-modes are trapped in the surface region of the stars. The effects of scattering and the refraction of sound waves by inhomogeneous turbulence elements (granules) cause serious energy loss and the additional phase shift of resonant sound waves, thus destroying the coherence of resonant sound waves, and limiting the coherence time of high-frequency p-modes (Gough, 1979). Until now we have had no reliable theory to treat and to estimate these damping effects due to the scattering and refraction of sound waves by turbulent elements, so they have been neglected in our theoretical calculations of non-adiabatic oscillations. The pulsation amplitude growth rate of highfrequency p-modes is overestimated by us, so our results for non-adiabatic oscillations are not as reliable for high-frequency modes, and we should be very cautious about them. For example, our theoretical calculation predicts that all solar p-modes with periods from 3 to $16 \mathrm{~min}$ (or frequencies from 1,050 to 5,500 $\mu \mathrm{Hz}$ ) are unstable. Our theory cannot explain the observed extreme linewidths of the high-frequency modes. So, we think that the high-frequency modes of the Sun should be stable. Toward high luminosity, the unstable modes shift to low-order modes which are trapped in the deeper interior region of stars. In comparison with excitation and damping due to the radiative $\kappa$-mechanism and convective coupling in the deep interior, the damping effects due to the scattering and refraction of sound waves, which mainly occur in the surface region, will weaken, and can even be negligible. Our theoretical results for non-adiabatic oscillations are in good agreement with observed mode instability from classic variables to high-luminosity red-giants (Figures 4-6, 8). However, our non-adiabatic oscillation equations are not applicable to the study of solar-like oscillators, because damping due to the scattering and refraction of sound waves by inhomogeneous turbulent elements has been neglected in our non-adiabatic pulsation equations. Expanding the applicable range of our non-adiabatic pulsation equations to cover solarlike oscillators and low-luminosity red giants would require the development of a theory for treating this damping effect of wave scattering and refraction by inhomogeneous turbulent elements.

Figure 1 shows the cumulative work $W$ and its components of the gas pressure $\left(W_{p g}\right)$, turbulent pressure $\left(W_{p t}\right)$, and turbulent viscosity $\left(W_{v i s}\right)$, as functions of depth $(\log T)$ for a low-luminosity $1.8 M_{\odot}$ red giant located at the lower part of the red-giant branch in the H-R diagram. Its low-order modes (panel a) are stable, while its high-order modes are unstable (panel b). Figure $\mathbf{2}$ is the cumulative work diagram, and is the same as Figure 1, but for a high-luminosity $1.8 M_{\odot}$ red giant. A few low-order modes are unstable (panel a), while all of the high-order modes $(n>4)$ are stable (panel b), as is typical for a Mira-like star. The component of gas pressure $W_{p g}$ includes the contributions of both the radiative and the convective fluxes. Using Eqs. 9-13, these two contributions can be separated. Figure 3 shows $W_{p g}$ and its components $\left(W_{L r}\right.$, $W_{L c}$, and $\left.W_{t h}\right)$ as functions of $\log T$. It can be seen that the component of convective enthalpy flux $W_{L c}$ is indeed a damping mechanism, which is the main factor for stabilizing low-order modes and defining the red edge of the Cepheid and Cepheid-like instability strips. Turbulent viscosity is the main mechanism stabilizing high-order modes.

Therefore, it can be found from the above analysis that the variable red giants of high luminosity are self-exciting. The excitation and stabilization of the oscillations result from the combined effects of the radiative $\kappa$-mechanism and the coupling between convection and oscillations. Turbulent pressure plays a 
significant role in the excitation of variable red giants. This may also be true for the intermediate-luminosity red giants.

\section{SUMMARY AND DISCUSSION}

Convection is an important and outstanding problem in astrophysics. Collectively, we have studied this subject for over fifty years. Our goal is to develop a stellar convection theory that is exact enough and simple enough in order to improve the treatment of overshooting in the calculations of stellar structure and evolution as well as the treatment of coupling between convection and oscillations in the theoretical calculations of stellar oscillations. In the present papers (including Parts I and II), a brief description of a time-dependent theory of convection developed by us (in Part I) was presented, and the progress of its application in the theoretical calculations of stellar structure and evolution (in Part I) and of stellar oscillations (in Part II) was reviewed. Our theoretical results achieve obvious improvements over the MLT formulation, which can be summarized as follows:

(1) Structure of the solar convection zone: Using our non-local convection theory, we calculated a model of the entire convective envelope of the Sun and studied the structure of both the upper and lower overshooting zones in detail (in Part I; Unno et al., 1985; Xiong and Cheng, 1992; Xiong and Deng, 2001; Deng et al., 2006). The results show that turbulent velocity and temperature fluctuations penetrate deeply into the convectively stable zone and decrease exponentially with $\ln P$ (Figure 4 in Part I). The e-folding length is about $1.4 \sqrt{C_{1} C_{2}}$ times the pressure scale height. Compared to the auto-correlations of turbulent velocity and temperature ( $x^{2}$ and $Z$ ), the penetration of convective flux $\mathrm{Fc}$ is smaller, because the convective energy transport is inefficient (the Peclet number $\mathrm{Pe}=x / x_{c}<\sim 1$ ) in the upper convective and overshooting zones, and the correlation coefficient of turbulent velocity and temperature decreases quickly $\left(\operatorname{Re}=\mathrm{V} / x Z^{1 / 2} \ll 1\right)$ toward the bottom of the convective zone. Passing through the boundary into the convectively stable zone, the convective flux changes its sign. These theoretical expectations agree well with the observations of the turbulent velocity temperature fields in the solar atmosphere (Leighton et al., 1962; Kell and Canfield, 1978; Nasis and Mattig, 1989; Komm et al., 1991). An obvious difference of our non-local model from the standard solar model (namely the local MLT) or non-local MLT models (Spiegel, 1963; Ulrich, 1970; Travis and Matsushima, 1973) is the presence of a larger temperature gradient at the top of the convective envelope compared to the other versions of MLT, because the convective flux becomes smaller at the top of the convective zone and becomes negative in the overshooting zone due to nonlocal convective diffusion. This steeper temperature profile agrees very well with the Harvard-Smithsonian reference atmosphere (Gingerich et al., 1971). The structure of the bottom convective-overshooting zone in our non-local convection theory also differs greatly from those of the other local or non-local MLT models. The temperature gradient has already become sub-adiabatic $\left(\nabla<\nabla_{a d}\right)$ in the convectively unstable zone before arriving at the bottom boundary of the convection zone. In the overshooting zone, the temperature gradient is subadiabatic and super-radiative $\left(\nabla_{r a d}<\nabla<\nabla_{a d}\right)$, because the convective flux is negative in the overshooting zone. Below the overshooting zone, the temperature gradient gradually approaches the radiative one (Figure $10 \mathrm{~B}$ in Part I). Therefore, we concluded that the sudden increase of sound speed at the bottom of the convective zone in the helioseismic sound-speed inversion (Christensen-Dalsgaard et al., 1996) is overestimated. This increase is not due to gravitational diffusion (Richard et al., 1996; Brun et al., 1999; Christensen-Dalsgaard and Di Mauro, 2007), but is an indication of non-local convective overshooting. Our theoretical predictions for the structure of the bottom convection and overshooting zones of the Sun outlined above (Xiong and Deng, 2001; Deng et al., 2006) agree well with the helioseismic sound-speed inversion. The relative differences in squared sound speed between our non-local model and the Sun are reduced greatly in comparison with the MLT model (Figure 5 in Part I; Zhang and Li, 2012; Zhang et al., 2012).

(2) Overshooting mixing and the evolution of massive stars: By using our non-local convection theory in chemically inhomogeneous stars (Xiong, 1981), we calculated the evolution of massive stars through the hydrogen burning stage (Xiong, 1986). The so-called semi-convection contradiction (Schwarzchild and Harm, 1958) is removed automatically, as predicted by us. The convectively neutral stability follows neither the Schwarzschild criterion nor that of Ledoux. $F_{C}=0$ is a more convenient and reasonable definition for the boundary of the convectively unstable zone. A molecular-weight gradient region adjacent to the convective core is formed automatically. Uncertainty of the semi-convection zone structure Stothers (1991), therefore of the evolution of massive stars, is removed automatically. The convective core increases due to non-local overshooting mixing, so the evolutionary track runs at a higher luminosity for a star with fixed mass, the main sequence band becomes noticeably wider and the main sequence lifetimes become longer in comparison with those calculated using the local MLT (Maeder, 1981). These influences of non-local convective mixing on the structure and evolution of stars increase toward lower mass, because the relative increase of convective core size increases with the decreasing mass of the star. These theoretical predictions have been confirmed by later theoretical studies (Stothers, 1991; Chiosi et al., 1992; Schootemeijer et al., 2019; Higgins and Vink, 2020). This means that the evolution masses for given luminosity is reduced in comparison with those calculated using the local MLT, for which the convective overshooting mixing has been neglected. This might be one of the important causes for the Cepheid mass discrepancy (Christy, 1968; Stobie, 1969; Cogan, 1970; Rodgers, 1970) and the contradiction between the theoretical and observed 
distribution of luminous stars in the $\mathrm{H}-\mathrm{R}$ diagram (Humphreys, 1978; Humphreys and Davidson, 1979). The Cepheid mass discrepancy between the new evolutionary masses calculated by an improve treatment of overshooting mixing, and the pulsation masses was resolved partially through improvements in the Baade-Wesselink method, as well as through better calibrations of the luminosities and of the effective temperatures (Chiosi et al., 1992; Gieren, 1988; Guzik et al., 2020). The theoretical calculations using the new, improved equation of state (Däppen et al., 1988; Hummer and Mihalas, 1988; Mihalas et al., 1988) and the new opacities (Rogers and Iglesias, 1992) show that the period ratios $\mathrm{P} 1 / \mathrm{P}$ (and $\mathrm{P} 2 / \mathrm{P} 0$ ) of beat Cepheids are, in general, reduced compared to those with the old Los Alamos Opacities (Cox and Tabor, 1976). This implies that the bump and beat Cepheid masses are increased. The inconsistency of the Cepheid bump-, and beat-mass with the evolutionary masses are also alleviated (Moskalik et al., 1992; Petersen, 1992; Simon, 1995; Keller and Wood, 2002; Buchler and Szabo, 2007). Therefore, the Cepheid mass discrepancies are partially resolved.

(3) Lithium depletion in stellar atmospheres: By using our nonlocal convection theory in chemically inhomogeneous stars (Xiong, 1981), we calculated the lithium depletion in the atmosphere of the Sun and late-type dwarfs (in Part I). The theoretical predictions agree well with observations of lithium abundances in the atmosphere of the Sun (Figures 9, 11 in Part I; Xiong and Deng, 2002) and of member stars of Galactic open clusters of different ages (Figure 11 in Part I; Xiong and Deng, 2009).

(4) Pulsational stability of stars: Linearizing the radiationhydrodynamic equations for the calculation of stellar structure and oscillations in Eqs. (2), $\left(3^{\prime}\right),\left(4^{\prime}\right),(5)$, and (18-21) in Part I, we can obtain a set of linear equations for radial and non-radial non-adiabatic oscillations. The radiative $\kappa$-mechanism and the thermodynamic- (through the convective enthalpy flux) and dynamic- (through the turbulent Reynolds Stress, i.e., turbulent pressure and turbulent viscosity) coupling between convection and oscillations have been taken into account in a selfconsistent way. With a slight modification, the $\varepsilon$-mechanism can be also included, if it is needed. Apart from damping due to the scattering and refraction of sound waves by inhomogeneous turbulent elements and stochastic excitation, they cover almost all the excitation and damping mechanisms for classical variables and variable red giants. By using our non-adiabatic oscillation equations and a fixed set of convective parameters $\left(C_{1}, C_{2} / C_{1}, C_{3}\right)=$ $(0.70,0.50,3.0)$ calibrated against the Sun, we carried out a linear stability analysis of radial and non-radial nonadiabatic oscillations for evolutionary models from the MS to RG phase for $1-20 M_{\odot}$ stars with solar abundance $(\mathrm{Y}=$ $0.26, Z=0.017)$. Almost all of the instability strips of the classical pulsating variables and variable red giants (Figures 4, 5), including the Cepheid, $\delta$ Scuti, $\gamma$ Doradus (Xiong et al., 2016), $\beta$ Cephei (Deng and Xiong, 2001), SPB, (Xiong and Deng, 2011), LPV, and OSARG (2018) were reproduced.
(5) Excitation and damping mechanisms of red giants were studied in detail: They are excited by the combined effects of the $к$-mechanism and coupling between convection and oscillations. Convective flux and turbulent viscosity are found to always have a damping effect. The damping effect of the convective flux is inversely proportional to the mode frequency, so it plays an important role in stabilizing the low-order modes and in defining the red edge of the instability strip. The damping effect of turbulent viscosity reaches its maximum at the point in the star where $3 \omega \tau_{c} / 16 \sim 1 ; \tau_{c}$ is the time scale of turbulent convection and $\omega$ is the angular frequency of the modes. Therefore, turbulent viscosity is the main damping mechanism for stabilizing the highorder modes of low-temperature stars. The turbulent pressure is, in general, an excitation mechanism; it reaches maximum at $3 \omega \tau_{c} / 4 \sim 1$. It plays an important role in the excitation of variable red giants (Figures 4-6, 8). The relative importance of the excitation and damping effects of turbulent pressure, turbulent viscosity, and convective enthalpy flux changes with the variation of stellar parameters, $\mathrm{M}, \mathrm{L}$, and Te, and the frequency (or the order/degree) of the mode. Our theory predicts that the low-order modes are stable, while the high-order modes are unstable for low-luminosity RGs. Toward high-luminosity, the unstable modes move gradually toward low-order, and all the intermediate- and high-order modes become stable, while a few low-order modes become unstable for high-luminosity red giants. These correspond to Mira-like stars (Figure 7). The frequency of the maximally unstable mode predicted by our theory is similar to that found using the scaling relation for the frequency of maximum amplitude of stochastically excited modes (Figure 10). So, it is impossible to distinguish these two excitation mechanisms using only the frequency of the maximum mode amplitude.

(6) The pulsation amplitude growth rates of high-frequency p-modes, which are high-order radial/non-radial modes trapped in the surface region of a star, are overestimated by our theory for low-luminosity low-temperature stars, because up to now we have had no reliable theory to treat and estimate the damping effect due to the scattering and refraction of sound waves by inhomogeneous turbulent elements and neglected it in our theoretical calculations of non-adiabatic oscillations. Our theoretical results for non-adiabatic oscillations are not as reliable for high-frequency p-modes of low-luminosity lowtemperature stars, so we should be very cautious about interpreting them. However, our theoretical results for nonadiabatic oscillations are reliable for the classical variables and high-luminosity red giants, because these have low-order modes trapped in the deep interior of the stars that are only lightly influenced by this damping. This inference has been supported by the fact that our theoretical results of non-adiabatic oscillations are in good agreement with observed mode instability from classic variables to high-luminosity red giants (Figures 4-6, 8). This may be also true for intermediateluminosity red giants. Our prediction that low-luminosity red giants might exhibit unstable intermediate-order modes still needs confirmation. It is also an uncertain prediction since we 


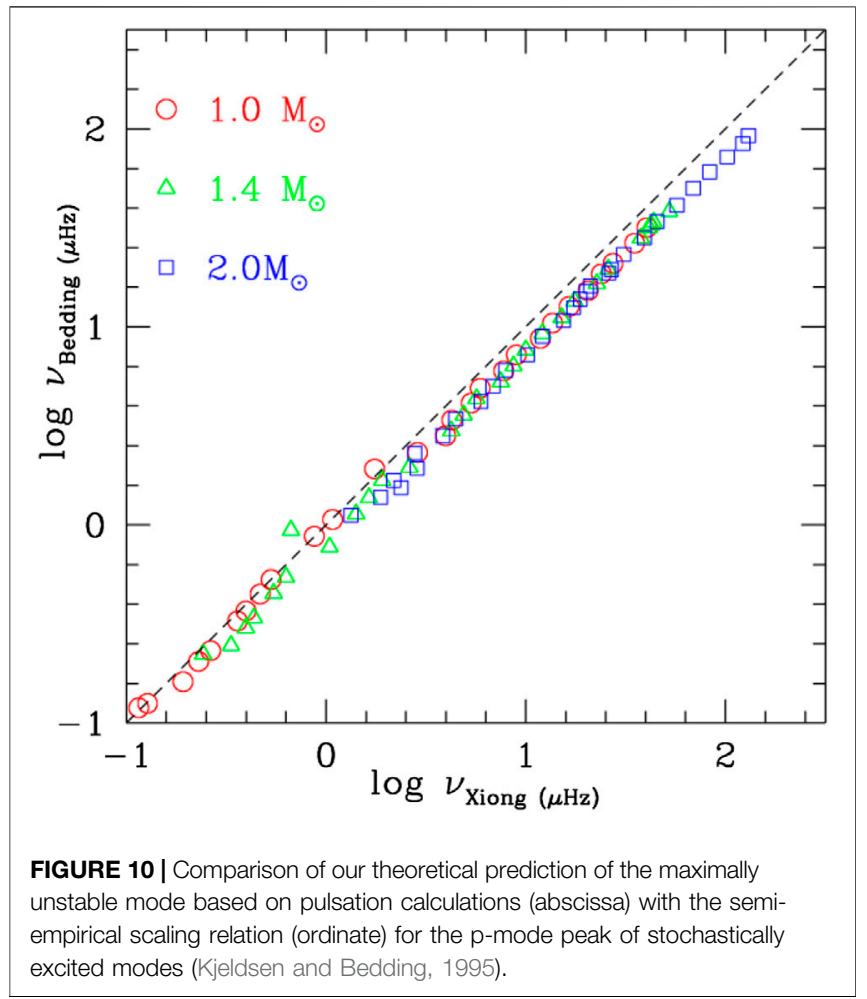

have not yet determined the range of stellar parameters for which our approach (neglecting the damping effects due to wave scattering and refraction by inhomogeneous turbulent elements) is valid. The ultimate solution to this problem depends on developing a theory for treating the damping effects due to the scattering and refraction of sound waves by inhomogeneous turbulent elements. This problem can be resolved in the not-too-distant future.

It is necessary to emphasize the following two points:

(1) The successes mentioned above do not only apply to an individual problem, but our theory is successful for many important problems in a wide field of stellar structure, evolution, and oscillations.

(2) Our non-local and time-dependent theory of convection includes three adjustable parameters of convection $\left(C_{1}, C_{2} / C_{1}, C_{3}\right)$, which are introduced in the modeling of the turbulent dissipation, turbulent diffusion, and turbulent anisotropy. They were calibrated by using the comparison between observations and the theoretical prediction for the depth and T-P structure of the solar convection zone, for the turbulence velocity-temperature fields in the atmosphere of the Sun, stellar evolution, lithium depletion in the atmosphere of the Sun and late-type dwarfs, and 3D simulations (Deng et al., 2006). The successes in the theoretical calculations of stellar structure, evolution, and oscillations mentioned above do not rely on the method of parameter adjustment. Our theoretical results, in general, are not sensitive to the convective parameters used. Except for the lithium depletion in the atmosphere of late- type dwarfs, all of the theoretical results for stellar structure, evolution, and oscillations are obtained using the same set of convective parameters calibrated against the Sun. The overshooting mixing is only slightly sensitive to the convective parameters used. It is necessary to make a small adjustment to the convective parameters with variation of the fundamental parameters of stars in the theoretical calculations of lithium depletion in atmospheres of late-type dwarfs.

All the successes mentioned above show that our dynamic theory of convection is roughly correct. It describes the dynamic behavior of turbulent convection more accurately than the MLT calculations. However, there are some difficulties in the calculations of the structure of the convective envelope for yellow giants and supergiants with $\mathrm{Te} \sim 6000-7000 \mathrm{~K}$ and for high-luminosity red giants. The evolutionary calculations with our non-local convection theory have been successful only for the hydrogen-burning stage of massive stars with initial mass $\mathrm{M}>$ $5 \mathrm{M}_{\odot}$. However, the evolutionary calculations cannot reach convergence for intermediate-mass stars with initial mass $M<$ $5 \mathrm{M}_{\odot}$ or for late stages of stellar evolution. The causes are not completely clear. These problems may result from the following two factors:

(1) An inadequate numerical method for converging the calculations

(2) Inadequacies of the convection theory

In the derivation of our convection theory, it was assumed that the turbulence is quasi-isotropic and the relative fluctuations of temperature and density are much less than 1 . So, our convection theory is applicable only for extensive (the scale of the convection zone is much larger than one pressure scale height) and subsonic convection. The convection zone becomes somewhat narrow in the surface convection zone of yellow giants and in the convective cores of intermediate-mass stars as well as at late evolutionary stages. In surface convection zones, the convective velocity increases with increasing stellar luminosity. Convection becomes nearly sonic or even locally supersonic for luminous red giants and supergiants. So, it is not difficult to understand why the convergence of numerical calculations would become difficult in the cases mentioned above, in which convection conditions deviate from quasiisotropic or become locally supersonic.

In comparison with MLT, our theory has obvious advantages for the treatment of the dynamic problems of non-local and timedependent convection. Its weaknesses are a lack of straightforwardness in the physical picture and the fact that the application becomes more complex in comparison with MLT. So, it is difficult to popularize. Up to now, it was only used by a small number of members within our own group and collaborators. There are many remaining problems to be addressed in the theory itself and its practicality. It is impossible to have a perfect convection theory until a better understanding of turbulence has been achieved. Our non-local and time-dependent theory is only one among numerous theoretical approaches for turbulent convection. The existence of different viewpoints and contention are very normal 
and helpful for the development of science. The applications of our theory in calculations of stellar convective envelope structure and oscillations have achieved some progress. However, more advances are necessary for the treatment of overshooting mixing in the late stages of stellar evolution using a completely non-local convection theory. We expect that more astronomers, hydrodynamicists, and applied mathematicians will join the collaborations for the research of convection theory and relevant problems.

\section{DATA AVAILABILITY STATEMENT}

The original contributions presented in the study are included in the article/Supplementary Material, further inquiries can be directed to the corresponding author.

\section{REFERENCES}

Aerts, C., Christensen-Dalsgaard, J., and Kurtz, D. W. (2010). Astroseismology. Berlin, Germany: Springer.

Alexander, D. R., and Ferguson, J. W., (1994). Low-temperature rosseland opacities. Astrophys. J. 437, 879. doi:10.1086/175039

Baker, N. H., and Gough, D. O. (1979). Pulsations of model RR Lyrae stars. Astrophys. J. 234, 232. doi:10.1086/157492

Balona, A., and Dziembowski, W. A. (2011). Kepler observations of $\delta$ Scuti stars. Mon. Not. Roy. Astron. Soc. 417, 591-601. doi:10.1111/j.1365-2966.2011.19301.x

Balona, L. A., Guzik, J. A., Uytterhoeven, K., Smith, J. C., Tenenbaum, P., and Twicken, J. D. (2011). The Kepler view of $\gamma$ Doradus stars. Mon. Not. Roy. Astron. Soc. 415, 3531-3538. doi:10.1111/j.1365-2966.2011.18973.x

Bertelli, G., Girardi, L., Marigo, P., and Nasi, E. (2008). Scaled solar tracks and isochrones in a large region of the Z-Y plane. Astron. Astrophys. 484, 815-830. doi:10.1051/0004-6361:20079165

Bertelli, G., Nasi, E., Girardi, L., and Marigo, P. (2009). Scaled solar tracks and isochrones in a large region of the Z-Y plane. Astron. Astrophys. 508, 355-369. doi:10.1051/0004-6361/200912093

Brun, A. S., Turck-Chieze, S., and Zahn, J. P. (1999). Standard solar models in the light of new helioseismic constraints. ii. mixing below the convective zone. Astrophys. J. 525, 1032-1041. doi:10.1086/307932

Buchler, J. R., and Szabo, R. (2007). Beat cepheids as probes of stellar and galactic metallicity. Astrophys. J. 660, 723-731. doi:10.1086/513071

Bohm-Vitense, T. R., and Stothers, R. B. (1958). Über die Wasserstoffkonvektionszone in Sternen Verschiedener Effektivtemperaturen und Leuchtkräfte. Mit 5 Textabbildungen, Z. Astrophys. 46, 108.

Chandrasekhar, S. (1939). An introduction to the study of stellar structure. Chicago, IL: University of Chicago Press.

Chiosi, C., Wood, P. R., Bertelli, G., Bressan, A., and Mateo, M. (1992). On the mass discrepancy of the Cepheid stars. Astrophys. J. 385, 205-216.

Christensen-Dalsgaard, J., Däppen, W., Ajukov, S. V., Anderson, E. R., Antia, H. M., Basu, S., et al. (1996). The current state of solar modeling. Science 272, 1286-1292. doi:10.1126/science.272.5266.1286

Christensen-Dalsgaard, J., and Di Mauro, M. P. (2007). Stellar evolution and seismic tools for asteroseismology-diffusive processes in stars and seismic analysis. Editors C. W. Straka, Y. Lebreton, and M. J. P. G. Monteiro (Les Ulis, France: EDP Sciences), 26.

Christy, R. F. (1968). Bump Cepheid models. Q. J. Roy. Astron. Soc. 9, 13.

Cogan, B. C. (1970). Pulsation constants for models of cepheids. Astrophys. J. 162, 139.

Cox, J. P., and Tabor, J. E. (1976). Radiative opacity tables for 40 stellar mixtures. ApJS 31, 271.

Cox, J. P. (1980). Theory of stellar pulsation. Princeton NJ: Princeton University press.

\section{AUTHOR CONTRIBUTIONS}

The author confirms being the sole contributor of this work and has approved it for publication.

\section{ACKNOWLEDGMENTS}

This work was supported by the National Nature Science Foundation of China (NSFC) through Grant No. 1137069. We would like to thank L. Deng and Dr C. Zhang for their valuable discussion. We thank Guzik and reviewer 3 for their kind help in revising the paper. I dedicate this article to my mentor, Prof. Wasaburo Unno. I thank him very much for his attention and support for our work over the years.

Däppen, W., Mihalas, D., Hummer, D. G., and Mihalas, B. W. (1988). The equation of state for stellar envelopes. III-thermodynamic quantities. Astrophys. J. 332, 261-270. doi:10.1086/166650

Deng, L., and Xiong, D. R. (2001). The $\beta$ Cephei instability strip. Mon. Not. $R$. Astron. Soc. 327, 881.

Deng, L., Xiong, D. R., and Chan, K. L. (2006). An anisotropic nonlocal convection theory. Astrophys. J. 643, 426-437. doi:10.1086/502707

Dupret, M-A., Grigahcene, A., Garrido, R., Gabriel, M., and Scuflaire, R. (2005). Convection-pulsation coupling-II. Excitation and stabilization mechanisms in $\delta$ Sct and $\gamma$ Dor stars. Astron. Astrophys. 435, 927. doi:10.1051/0004-6361:20041817

Eggen, O. J. (1977). The classification of instrinsic variables VII. The medium -amplitude red variables. ApJ 213, 767.

Eggen, O. J. (1972a). The classification of intrinsic variable stars. 1. The Red variables of type N. Astrophys. J. 174, 45-55. doi:10.1086/151467

Eggen, O. J. (1972b). The classification of intrinsic variable stars. II. The red variables of S and related types. Astrophys. J. 177, 489-507.

Eggen, O. J. (1973a). The classification of intrinsic variable. III. Calibration of the luminosities of small amplitude red variables in the old disk population. Astrophys. J. 180, 857-870. doi:10.1086/152012

Eggen, O. J. (1973b). The classification of intrinsic variables. IV. Very-smallamplitude, very-short-period red variables. Astrophys. J. 184, 793-799.

Eggen, O. J. (1975). The classification of intrinsic variables. V. The large-amplitude variables. Astrophys. J. 195, 661-678.

Gieren, W. P. (1988). The Galactic Cepheid period-luminosity relation from the visual surface brightness method. Astrophys. J. 329, 790-796.

Gingerich, O., Noyes, R. W., Kalkofen, W., and Cuny, Y. (1971). The HarvardSmithsonian reference atmosphere. Sol. Phys. 18, 347-365. doi:10.1007/ BF00149057

Gonczi, G., and Osaki, Y. (1980). On local theory of time-dependent convection in the stellar pulsation problem. A\&A. 84, 304.

Gough, D. O. (1979). Some theoretical remarks on solar oscillations, in Nonradial and Nonlinear Stellar Pulsation. Berlin, Germany: SpringerVerlog, p. 273.

Grigahcene, A., Antoci, V., Balona, L., Catanzaro, G., Daszyńska-Daszkiewicz, J., Guzik, J. A., et al. (2010). Hybrid $\gamma$ doradus- $\delta$ Scuti pulsators: new insights into the Physics of the oscillations from kepler observations. Astrophys. J. 713, 192-197. doi:10.1088/2041-8205/713/2/L192

Guzik, J. A., Farag, E., and Ostrowski, J. (2020). Investigating opacity modifications and reaction rate uncertainties to resolve the cepheid mass discrepancy. arxiv. doi:10.204073G2020/62

Guzik, J. A., Kaye, A. B., Bradley, P. A., Cox, A. N., and Neuforge, C. (2000). Driving the gravity-mode pulsations in $\gamma$ Doradus variables. Astrophys. J. 542, 57.

Hareter, M. (2012). Preliminary results on $\gamma$ Dor and $\delta$ Sct $\gamma$ Dor hybrids in the CoRoT exo field of LRa01. Astron. Nachr. 333, 1048-1050. doi:10.1002/asna. 201211790 
Higgins, E. R., and Vink, J. S. (2020). Theoretical investigation of the Humphreys-Davidson limit at high and low metallicity. Astron. Astrophys. 635, 175-186. doi:10.1051/0004-6361/201937374

Hinze, J. O. (1975). Turbulence. New York: McGraw-Hill.

Houdek, G. (2000). Delta Scuti and related stars. Editors M. Breger and M. H. Montgomery (San Francisco: Astronomical Society Pacific), 210, 454.

Hummer, D. G., and Mihalas, D. (1988). The equation of state for stellar envelopes. I. An occupation probability formalism for the truncation of internal partition functions. Astrophys. J. 331, 794. doi:10.1086/166600

Humphreys, R. (1978). Studies of luminous stars in nearby galaxies. I. Supergiants and $O$ stars in the Milky Way, 38, 309.

Humphreys, R., and Davidson, K. (1979). Studies of luminous stars in nearby galaxies. III-Comments on the evolution of the most massive stars in the Milky Way and the Large Magellanic Cloud. Astrophys. J. 232, 409-420.

Keel, S. L., and Canfield, R. C. (1978). The height variation of velocity and temperature fluctuations in the solar photosphere. Astron. Astrophys. 70, 169-179.

Keeley, D. A. (1977). Linear stability analysis of stellar models by the inverse iteration method. ApJ. 211, 926.

Keller, S. C., and Wood, P. C. (2002). Large magellanic cloud bump cepheids: probing the stellar mass-luminosity relation. Astrophys. J. 578, 144-150.

Kjeldsen, H., and Bedding, T. R. (1995). Amplitudes, of stellar oscillations: the implications for asteroseismology. A\&A. 293, 87.

Komm, R., Mattig, R. W., and Nesis, A. (1991). The small-scale velocity field in the solar photosphere. Astron. Astrophys. 243, 251-262.

Ledoux, P., and Walraven, T. (1958). Handbuch der physic. Editor S. Flugg (Berlin: Springer-Verlag), Vol. 51, 353.

Leighton, R. B., Noyes, R. W., and Simon, G. W. (1962). Velocity fields in the solar atmosphere. I. Preliminary report. Astrophys. J. 135, 474.

Madore, B. F. (1982). The period-luminosity relation. IV-Intrinsic relations and reddenings for the Large Magellanic Cloud Cepheids. Astrophys. J. 253, 575-579.

Maeder, B. F. (1981). Grids of evolutionary models for the upper part of HR diagram. Mass loss and turning of some red supergiants into WR Stars. A\&A. $102,401$.

Mihalas, D., Däppen, W., and Hunmer, D. G. (1988). The equation of state for stellar envelopes. II-Algorithm and selected results. Astrophys. J. 331, 815-825.

Moskalik, P., Buchler, J. R., and Marom, M. N. (1992). Toward a resolution of the bump and beat Cepheid mass discrepancies. Astrophys. J. 385, 685-693.

Moskalik, P., and Dziembowski, W. A. (1992). New opacities and the origin of the Beta Cephei pulsation. Astron. Astrophys. 256, L5-L8.

Nasis, A., and Mattig, W. (1989). The height dependence of vertical and horizontal velocities attributed to the convective overshoot in the solar atmosphere. Astron. Astrophys. 221, 130-136.

Pamyatnykh, A. A. (2000). Delta Scuti and related stars. Editor M. Breger and M. H. Montgomery (San Francisco: Astronomical Society Pacific), 210, 215.

Petersen, J. O. (1992). Studies of Cepheid-type variability. X-Kappa-effectfunctions for period ratios. Astron. Astrophys. 265, 555-562.

Richard, O., Vauclair, S., Charbonnel, C., et al. (1996). New solar models including helioseismological constraints and light-element depletion. A\&A. 312, 1000.

Rodgers, A. W. (1970). The masses of pulsating stars. Mon. Not. Roy. Astron. Soc. 151, 133-140. doi:10.1093/mnras/151.1.133

Rogers, F. J., and Iglesias, C. A. (1992). Radiative atomic Rosseland mean opacity tables. Astrophys. J. Suppl. Ser. 79, 507-568.

Sarro, L. M., Debosscher, J., Neiner, C., Bello-Garcia, A., González-Marcos, A., Prendes-Gero, B., et al. (2013). Improved variability classification of CoRoT targets with Giraffe spectra. Astron. Astrophys. 550, 120. doi:10.1051/0004$6361 / 201220184$

Schootemeijer, A., Nanger, N., and Grin, N. J. (2019). Constraining mixing in massive stars in the small magellanic cloud. A\&A 625A, 132.

Schwarzscild, M., and Harm, R. (1958). Evolution of very massive stars. Astrophys. J. 128, 348. doi:10.1086/146548

Simon, N. R. (1995). ASPC series. Editors S. J. Adelman and W. L. Weise (San Francisco: Astronomical Society Pacific), Vol. 78, 211.

Soszynski, I., Dziembowski, W. A., Udalski, A., Kubaik, M. K., Szymanski, G., Pietrzynski, L., et al. (2007). The optical gravitational lensing experiment. Periodluminosity relations of variable red giant stars. Acta Astronom. 57, 201-225.
Soszynski, I., Udalski, A., Szymansi, M. K., Kubiak, M., et al. (2009). The optical gravitational lensing experiment. The OGLE-III catalog of variable stars. IV. Long-period variables in the large magellanic cloud. Acta Astronom. 59, 239-253.

Soszynski, I., Udalski, A., Kubiak, M., Szymanski, M. K., Pietrzynski, G., Zebrun, K., et al. (2005). The optical gravitational lensing experiment. Miras and semiregular variables in the Large Magellanic Cloud. Acta Astronom. 55, 331-348.

Soszynski, I., Udalski, A., Kubiak, M., Szymanski, M., Peitrzynski, G., Zebrun, K., et al. (2004). The Optical Gravitational Lensing Experiment. Small Amplitude Variable Red Giants in the Magellanic Clouds. Acta Astronom. 54, 129.

Spiegel, E. A. (1963). A generalization of the mixing-length theory of turbulent convection. Astrophys. J. 138, 216.

Stello, D., Bruntt, H., Kjeldsen, H., Bedding, T. R., Arentoft, T., Gilliland, R. L., et al. (2007). Multisite campaign on the open cluster M67-II. Evidence for solar-like oscillations in red giant stars. Mon. Not. Roy. Astron. Soc. 377, 584-594. doi:10. $1111 / j .1365-2966.2007 .11585 . x$

Stobie, R. S. (1969). Cepheid pulsation-I: numerical technique and test calculations. Mon. Not. Roy. Astron. Soc. 144, 461-484. doi:10.1093/mnras/144.4.461

Stothers, R. B. (1991). Observational tests of convective core overshooting in stars of intermediate to high mass in the Galaxy. Astrophys. J. 383, 820-836. doi:10.1086/170840

Takayama, I., Saio, H., and Ita, Y. (2013). On the pulsation modes of OGLE small amplitude red giant variables in the LMC. Mon. Not. Roy. Astron. Soc. 431, 3189-3195. doi:10.1093/mnras/stt398

Tian, B., Men, H., Deng, L., Xiong, D.-R., and Cao, H.-L. (2003). Statistics of the instability strip of Cephei stars. Chin. J. Astron. Astrophys. 2, 125-132. doi:10. 1088/1009-9271/3/2/125

Trabucchi, M., Wood, P. R., Montalbán, J., Marigo, P., Pastorelli, G., and Giradi, L. (2017). A new interpretation of the period-luminosity sequences of long-period variables. Astrophys. J. 847, 139. doi:10.3847/1538-4357/aa8998

Travis, L. D., and Matsushima, S. (1973). The role of CONVECT10N in stellar atmospheres. Observable effects of convection in the solar atmosphere. Astrophys. J. 180, 975-986. doi:10.1086/152020

Ulrich, R. K. (1970). The five-minute oscillations on the solar surface. Astrophys. J. 162, 993. doi:10.1086/150731

Unno, W., Kondo, M.-A., and Xiong, D. R. (1985). Solar convection zone given by nonlocal mixing-length theory. Publ. Astron. Soc. Jpn. 37, 235-244.

Unno, W., Osaki, Y., and Ando, H., (1989). Nonradial oscillations of stars. 2nd Edn. Tokyo: University of Tokyo Press.

Uytterhoeven, K., Moya, A., Grigahcene, A., Guzik, J. A., Gutiérrez-Soto, J., Smalley, B., et al. (2011). The Kepler characterization of the variability among A- and F-type stars. Astron. Astrophys. 534, 125. doi:10.1051/0004$6361 / 201117368$

Wood, P. R. (2000). Variable red giants in the LMC: pulsating stars and binaries? PASA, 17, 18

Wood, P. R. (2019). Modelling long-period variables-I. A new grid of O-rich and C-rich pulsation models. Mon. Not. Roy. Astron. Soc., 482, 929-949. doi:10. 1093/mnras/sty2745

Wood, P. R. (2015). Oscillatory convective modes in red giants: a possible explanation of the long secondary periods. Mon. Not. Roy. Astron. Soc., 448, 3829. doi:10.1093/mnras/stv1587

Xiong, D. R., and Deng, L. (2007). Non-adiabatic oscillations of red giants. MNRAS, $378,1270$.

Xiong, D. R., and Deng, L. (2009). Lithium depletion in late-type dwarfs. Mon. Not. Roy. Astron. Soc., 395, 2013-2028. doi:10.1111/j.1365-2966.2009.14581.x

Xiong, D. R. (1978). Statistical theory of turbulent convection in pulsating variables. ChA 2, 118.

Xiong, D. R. (1980). A statistical theory of non-local convection. ChA 4, 234.

Xiong, D. R. (1981). Statistical theory of non-local convection in chemically inhomogeneous stars. SciSn 24, 1406.

Xiong, D. R., Cheng, Q. L., and Deng, L. (1997). Nonlocal time-dependent convection theory. ApJS 108, 529.

Xiong, D. R., Cheng, Q. L., and Deng, L. (1998b). Turbulent convection and pulsational stability of variable stars. II. Oscillations of RR lyrae and horizontal branch red variable stars. Astrophys. J. 500, 465.

Xiong, D. R., and Deng, L. (2001). The structure of the solar convective overshooting zone. MNRAS 327, 1137.

Xiong, D. R., and Deng, L. (2002). The extent of the solar overshooting zone deduced from Li abundance. MNRAS 336, 511. 
Xiong, D. R., Deng, L., and Cheng, Q. L. (1998a). Turbulent convection and pulsational stability of variable stars. I. Oscillations of long-period variables. Astrophys. J. 499, 355.

Xiong, D. R., Deng, L., and Zhang, C. (2015). Mon. Not. Roy. Astron. Soc. 451, 3354.

Xiong, D. R., Deng, L., and Zhang, C. (2018). Mon. Not. Roy. Astron. Soc. 480, 2698.

Xiong, D. R., Deng, L., Zhang, C., and Wang, K. (2016). Turbulent convection and pulsation stability of stars-II. Theoretical instability strip for $\delta$ Scuti and $\gamma$ Doradus stars. Mon. Not. Roy. Astron. Soc. 457, 3163-3177. doi:10.1093/mnras/stw047

Xiong, D. R. (1986). The evolution of massive stars using a non-local theory of convection. Astron. Astrophys. 167, 239-246.

Xiong, D. R., and Cheng, Q. L. (1992). A note on the mixing length theory and massive star evolution. Chin. J. Astron. Astrophys. 254, 362.

Xiong, D. R., and Deng, L. (2011). Pulsationally unstable nonradial modes in upper main sequence. ACASn, 52, 115.

Xiong, D. R., and Deng, L. (2010). Non-adiabatic oscillations of the low- and intermediate-degree modes of the Sun. Mon. Not. Roy. Astron. Soc. 405, 2759-2767. doi:10.1111/j.1365-2966.2010.16662.x

Xiong, D. R., and Deng, L. (2013). Solar-like and Mira-like oscillations of stars-a uniform excitation mechanism. RAA 13, 1269.
Xiong, D. R. (1989). Radiation-hydrodynamic equations for stellar oscillations. Astron. Astrophys. 209, 126-134.

Zhang, C., Deng, L., Xiong, D., and Christensen-Dalsgaard, J. (2012). Sound-speed inversion of the Sun using a nonlocal statistical convection theory. Astrophys. J. Lett 759, L14. doi:10.1088/2041-8205/759/1/L14

Zhang, Q. S., and Li, Y. (2012). Turbulent convection model in the overshooting region. I. Effects of the convective mixing in the solar overshooting region. Astrophys. J. 746, 50. doi:10.1088/0004-637X/746/1/50

Conflict of Interest: The author declares that the research was conducted in the absence of any commercial or financial relationships that could be construed as a potential conflict of interest.

Copyright (c) 2021 Xiong. This is an open-access article distributed under the terms of the Creative Commons Attribution License (CC BY). The use, distribution or reproduction in other forums is permitted, provided the original author(s) and the copyright owner(s) are credited and that the original publication in this journal is cited, in accordance with accepted academic practice. No use, distribution or reproduction is permitted which does not comply with these terms. 